\title{
Review on the Fundamental Aspects of Petroleum Oil Emulsions and Techniques of Demulsification
}

\author{
Souleyman A Issaka*, Abdurahman H Nour and Rosli Mohd Yunus \\ Faculty of Chemical and Natural Resources Engineering, University Malaysia Pahang, Malaysia
}

\begin{abstract}
This review is aimed to introduce a comprehensive survey on the most prominent and sustainable techniques and methods that could abate the environmental worries as well as financial insecurities in treating petroleum emulsions, since the existence of water is not desired because of the paramount troubles it may cause on the processing streamlines, as well as financial cost associated with transporting water mixed with petroleum. Currently, the most commonly used method for treating petroleum emulsion is the application of chemical additives, known as demulsifiers. Althogh, there are many other methods that are claimed to be more favorable from economic and environmental perspectives, yet, have not being fully put into real life practice, because of the drawbacks and disadvantages. In this review, several techniques have been surveyed including, chemical, electrical, membrane, centrifuge, bacteria, air floatation, ultrasonic, and microwave. Based on this Theoretical survey, silcone based demulsifiers were reported to be very effective and environmental friendly, but expensive. Also microwave and ultrasonics were reported to be very effective in treating petroleum emulsion and could be recommended as future ulternatives for treating petroleum emulsions.
\end{abstract}

Keywords: Petroleum emulsions; Demulusification techniques; W/O emulsion separation; Stability

\section{Introduction}

This review paper would investigate the related literature and previous studies on the petroleum emulsions, and techniques of separating water from crude petroleum oils. since water separation from petroleum is vital from both tchnical as well as environmental view points.

\section{Emulsion formation and stability}

Basically all types of emulsions consist of two immiscible liquids, one of which is dispersed as droplets called internal phase or droplet phase) into another, that is normally called continuous phase. These two phases are also normally stabilized by a third phase, called emulsifying agent (also known as surfactants, surface active agent, or emulsifiers). In water-in-crude (w/o) emulsions, the dispersed water droplets (internal phase) are encapsulated by an oil matrix (external or continuous phase). Without the emulsifying agent, emulsions made of the individual pure components of only oil and water phases are not stable, given enough time, they would quickly split back into their original phases (water and oil). that is due to the elevated interfacial tension between the oil and water phases. High interfacial tension would induce the attractive forces to attact the neighboring water droplets, wich will soon merge together and eventually separate to two distict phases (water and oil). Therefore a surface active agent or emulsifier is needed to stabilize the interfacial films, and eventually the whole system. Naturally, petroleum crude oil emulsions are stabilized by the indigenous surfactants, namely asphaltenes and resins which are partially soluble in both systems and have a strong tendency to migrate and sediment at the interface between the water droplet and the oil phase forming what so called interfacial barrier [1]. However, most researchers use experimental emulsions, which is produced in laboratory via commercial serfactants to mimic the natural emulsion. Stable Water-in-crude Emulsions are generated during crude oil mining, because water and oil coexists in the resevoir, the come to contact and mix while pumped out on the wellbore, Indeed, the existence of the indigenous surfactants, as such asphaltenes and resins, heavy metals, fatty acids and clays would strongly lower the interfacial tension between the oil and water, and lead to the formation of stable barriers between the water-oil interface that resist the coalescence of water droplets and eventually produce stable water-in-crude oil emulsions [2,3]. Crude oil constitudes of various components, mainly, carbon, hydrogen. Minor quantities of others gases such as nitrogen, oxygen, together with heavy metals, such as sulfur, and trace amount of other common metals (iron, vanadium, and nickel). These elements would facilitate the formation of hydrogen bonding as well as polar interactions among the asphaltene components, and that would give the interfacial film its elastic behavior and strength [4]. Plenty of researches was conducted on the emulsion preparation and stabilization process in the field of crude oil emulsion. Various compositions, combinations, as well as preparation conditions, have been reported. since emulsions are multiphase systems of mainly Oil, water and emulsifying agent, that moulded together by the means of mechanical mixing, therefore stability study is aimed to identify the right variables, including, type of chemicals, mixing power and speed, mixing duration (emulsification time), mixing or emulsification temperature (EMT). Knowlege of these parameters is vital, in producing any type of stable experimental emulsion. Tron eric Havre and Johan sjoblom, have investigated the effectiveness of combined surfactants Phases and Asphaltene particles in producing stable emulsions. Solution of heptane/Toluene mixture, that contains Paraffinic and aromatic components was prepared to represent the crude oil phase. They extracted the asphaltene from an original crude, this asphaltene is then

*Corresponding author: Issaka SA, Faculty of Chemical and Natural Resources Engineering, University Malaysia Pahang, Malaysia, Tel: 00609-549-2501; Fax: 00609-549-2544; E-mail: sahame@gmail.com

Received March 09, 2015; Accepted March 16, 2015; Published March 23, 2015

Citation: Issaka SA, Nour AH, Yunus RM (2015) Review on the Fundamental Aspects of Petroleum Oil Emulsions and Techniques of Demulsification. J Pet Environ Biotechnol 6: 214. doi:10.4172/2157-7463.1000214

Copyright: @ 2015 Issaka SA, et al. This is an open-access article distributed under the terms of the Creative Commons Attribution License, which permits unrestricted use, distribution, and reproduction in any medium, provided the original author and source are credited. 
combined with aforementioned commercial surfactants phase to represent the emulsifying agent. Ultra Turrax T25 mixture was used at speed of $9500 \mathrm{rpm}$ for 30 seconds (emulsification time). Emulsification was brought about in water bath at $70^{\circ} \mathrm{C}$. Their prepared emulsion was investigated based on stability as well as droplet size analysis. Their study revealed that water-in-crude oil emulsions can be stabilized by means of combined surfactants as such naphthenic acid, naphthenate and asphaltene particles [5]. Lixin Xia also investigated the effect of asphaltene and resin in jet kerosene continuous phase w/o emulsion, but at different emulsification conditions, he used hand shaking as mechanical mixing source. He characterized his emulsions using optical microscopic visualization, and water resolution techniques. His result recomended the water resolution technique as the best way to study the stability of emulsions [6]. In another work Brujic, have produced a transparent Oil-in-water emulsion, the intended to measur the inter-droplet forces that govern these micron sized emulsion droplets. Silicone Oil and Sodium dodecyl Sulphate (SDS) were selected as oil phase and emulsifying agents respectively, Mixing speed was $7000 \mathrm{~s}^{-1}$, They produced emulsion with mean droplet size of $3.4 \mu \mathrm{m}$, ranging from $(1-10 \mu \mathrm{m})$, which was reported to be stable over a period of at least one year [7]. Guo et al., have investigated the significance of alkaline-surfactant-polymers as emulsifiers to stabilize emulsions made from model oil. Their model oil, consisted of jet fuel as the dispersing medium, mixed with fractions from Gudong crude oil (from Shengeli oil field in China) to mimic the natural crude oil composition. Emulsions were prepared via simple hand mixing at $60^{\circ} \mathrm{C}$. Emulsion stability was determined by visually monitoring the rate of water resolution. The interfacial active components in the Asphaltene were found to be ionized when they are mixed with the alkali solution, thus, the ionized and non ionized groups can make hydrogen bonds, which lead to the formation of the acidic soaps. These soaps act as emulsifiers and promote stable emulsion on petroleum wells during enhanced oil recovery where the alkaline-surfactant-copolymers are injected in the reservoir to increase the productivity [8]. Stability effect of the alkalinesurfactant-copolymer also investigated by Minguan et al. on stabilizing Daqing crude oil from China. They comfirmed the ability of alkali surfactants to produce stable emulsions. Model oil was prepared from jet fuel mixed with some polar fractions from daqing crude oil. The water to oil ratio was $50-50 \%(\mathrm{v} / \mathrm{v})$, emulsion was prepared by handshaking at $45^{\circ} \mathrm{C}$ [9]. Venezuelian crude oil having API of $24.5^{\circ}$ (sour crude oil), was found to produce be very stable emulsions, even without emulsifiers. Thus, in the subsequent studies, researchers have diluted the original Venezuelan crude oil with various amount of heptanes (Poor solvent for asphaltene), several emulsions were made from the diluted oil by vigorous mixing at $40^{\circ} \mathrm{C}$. results proved that the Alifatic/Aromatic ratio of the crude oil can tremendously affect the stability of the emulsion. This affirm the theory that attributing the asphaltene and resin activities to be strong function of the Alifatic/ aromatic fractions of the petroleum. Hence, when the heptane (alifatic) is added to the venezuelan crude oil (dilution), it was found to induce the asphaltene precipitation out of the crude oil. This precipitation increases the size and poly-dispersity of submicrone asphaltene particles that lead eventually to reduce the stability of the emulsion, because of the attractive forces [10]. Nave Aske et al., have developed an electric cell that can supply an electric field to the emulsion, it was used to predict emulsion stability, since the electric field can break the emulsion and separate the water. They have successfully tested that device on emulsions made from 21 different crude oils with different water cuts (30\% and $20 \%)$, and from their reported results, they have categorised the stability into several groups, mainly, stable and unstable, and that was imparted that to the asphaltene contain, its aggregation state and the interfacial elasticity [11]. In another work, bitumen was dispersed in water phase, and produced a stable bitumen-in-water emulsion at elevated $\mathrm{pH}$ values [12]. Minguan Li et al. have investigated the effects of alkaline surfactant-polymers on stabilizing crude oil emulsion that is originated from Daqing oil field (China), however, their results had proved the ability of theses Alkaline surfactant to produce stable w/o emulsions inside the reservoir when they are injected during enhanced oil recovery. Their sample was composed of 50-50\% (w/o), and prepared by simple hand shaking [9].

In another work, Andreas et al. have studied the effects of silica nano-particles on stabilizing model oil emulsions. Asphaltene and resins were extracted from the Brazilian sour crude oil and used as coating on the surface of the silica particles to render them amphiphilic solid emulsifiers. Heptanol-toluene mixture was choosen as model oil phase. ultraturax mixture was used at speed of $22000 \mathrm{rpm}$ for three minutes. The stability study was carried out within a period of 30 minutes. The percentage of water resolved from the emulsion within this 30 minutes was used to assess the emulsion stability. Their results showed that, coatings has greatly enhanced the model emulsion stability [13]. Elsewhere, the interfacial activity of asphaletene were measured, a model oil emulsions were prepared by mixing Heptane, Toluene, Asphaltene, Resin, and Native solids. The resulting emulsions were characterised by calculating the emulsion surface area from droplet sizes distribution. The Asphaltene and Resin used in their study were extracted from two different crude oils, although, their emulsion contained $40 \%$ water, mixed at $17000 \mathrm{rpm}$, for 7 minutes, yet it was observed to separate within few minutes form it is first preparation [12]. Far from crude oil emulsions, other researchers reported the polymer blind mixture. However, in comparision to emulsions, surfactants-like additives are used as stabilizers in polymer blends. theses additives are called Compatibilizers, and used to stabilize the non-compatible polymers. Moran et al., have studied the role of Naphthenic acid in crude oil emulsions, since naphthenic acid is one of the natural indigenous components of the crude oil. Thus, they have produced model oil (Heptane/toluene) emulsions that was stabilized by Naphthenic acid based surfactant, namely, Sodium Naphthenate, at different concentration ranges $(0-10 \%)$. sonicator was used to mix the emulsion sample [14]. Others, have investigated the effects of physicals parameters, including internal phase volume fractions, Mixing speed (rpm), mixing (emulsification temperature) on the rheological properties of water-in-crude oil emulsions [15]. Rosli Daike et al., studied the effect of types and concentrations of various commercial emulsifiers in stabilizing Liquid natural rubber (LNR) based w/o emulsions. Various concentration of emulsifiers were used (ranged from 1-6\%). Emulsions were prepared at room temperature with continuous stirring for 30 minutes. Their results proved that, Liquid Natural Rubber with lower molecular weight had produced emulsions with smaller droplet sizes, high viscosity and more stable emulsions. Whereas, the opposite pattern was observed with the lower molecular weight (LNR). In the same fashion, Adam Macierzanka et al. have investigated the microstructure of the internal phase of emulsions stabilized by Acylpropyleneglycols containing $\mathrm{C}_{16} / \mathrm{C}_{18}$ fatty acids. The oil phase was mixture of Paraffin oil and paraffin wax. Emulsions were prepared in a glass thermostate emulsor, mechanical two blade agitator was used at mixing speed of $500 \mathrm{rpm}$, the phase composition of the emulsions was varied from $20-80 \%$ (water-oil\% respectively). Lei Zhang and others, have studied the correlation between the Hydrophilic-Lipophilic Balance (HLB) of the surfactants and the emulsion properties. They used surfactants with various HLB numbers to prepare experimental water-in-oil emulsions, two blade stirere was 
used at mixing speed of $500 \mathrm{rpm}$ for 15 minutes. Span 80 and Tween 80 were chosen to be the major stabilizers for their study. various other fractions with different HLB ranging from 4.5-15 were produced by blending [16]. Oppositely Svetlan et al. have reported a comprehensive investigation on emulsion Rheology, but focused their attentions mainly on the effect of the individual components on emulsion rheology of dilute emulsions of oil base and polymer blends [17]. Also Minguan Li et al. studied the stability of model emulsions that was stabilized by the indigenous incompatible components of the crude oil, thus the stability was investigated by means of various apparatus such as IR, UV, and GC-MS, hence the interfacial active components (Surfactants) were extracted from two different crude oils that originated from Saudi Arabia and Shengli (China). The interfacial active components (Asphaltene and resins) are normally extracted from the crude oils by SARA fractionation techniques. SARA is stand for Saturate, Aromatic, Resin, and Asphaltene, the main components in crude oil. In SARA extraction process, crude oil is first mixed with $\mathrm{n}$-hexane in the ratio of 1:30 (v/v) respectively. The mixture is left to settle for 24 hours. The precipitated asphaltene could be filtered out and dried. The percentage of asphaltene in the crude oil is calculated based on the wheight of this precipitated asphaltene. the n-hexane soluble part, is then poured in a silica column after evaporating the solvent to percolate the remaining SARA fractions by their respective dissolving solvents [18]. Beside asphaltenes and resins, stability of petroleum emulsions also reportedely, depends on combination of several factors such as molecular size of the interfacial active components, aromaticity or aromatic condensation of the crude oil itself, and carbonyl group concentrations [18]. Marco A. Farah, odserved the dependence of water-in-crude oil emulsions on the other physico-chemical properties of the system such as dispersed phase (water) volume fractions, temperature, average droplets size, shear rate, droplets sizes distributions, viscosity and density of the of the oil. They varied the water content of the emulsion as $10,20,30,40$, and $60 \%$. Mixing speed was fixed at $10,000 \mathrm{rpm}$ for three minutes. Stability pattern was verified by measuring the amount of water resolved from the stable emulsion within settling duration of four hours [19]. In emulsion field, every new emulsion sample of what ever application, has to undergo some stability test before proceeding to its ultimate application. It was observed that, water-in-crude oil emulsions of high API ( $\geq 38$ ) crude oils, was very difficult to produce stable emulsions especially at high water volume fractions. Also increasing the water volume fraction in emulsion would increase the temperature at which emulsion shows Newtonian behavior. Below wax appearance temperature (WAT), emulsions present rheological behavior of a bingham plastic in certain shear range, indeed variation of kinematic viscosity of the emulsion with temperature was observed to correspond with the ASTM equation [19]. Sanfeld and others, investigated the repulsive interaction within droplets of dense w/o emulsions, and found that, the thickness of the repulsive electrical double layer is inversely proportional to the internal phase (water) Volume fractions [20]. Indeed Kristofer Paso et al., have observed the stability as well as flow properties of two Brazilian crude oils. Their emulsions were composed of 30,50 , and $70 \%$ water volume fractions. Ultra-turax homogenizer was used for mixing at $24000 \mathrm{rpm}$ for 2 minutes. Emulsification temperature also were varied as 4,25 and $60^{\circ} \mathrm{C}$. They concluded that at low water volume fractions, emulsion can resists coalescence in a better manner than its high water volume fraction counter part. Another observation was that, the waxy crude oil was able to form stable emulsion with water cut as high as $70 \%$, while the heavy oil forms stable emulsions with water cut as high as $50 \%$. This superior stability of the waxy crude oil might be attributed to the greater abundance of Asphaltene and lesser abundance of resins [21]. In another report, Christophe Dicharry et al., have investigated the viscoelastic character of the interfacial film at the water-oil interface by means of the oscillating drop tensiometer method. They concluded that, interfacial parameters such as interfacial tension and elasticity modulus, can help to predict emulsion stability. Stability was a found to increase proportionally with increasing the interfacial gel strength and glass transition temperature of the gel [22].

Einar Eng Johnsen et al. have had proposed a method for measuring the viscosity of emulsions under pressurized conditions, their apparatus that consists of rotating wheel that would make the fluid inside to move to the opposite direction, hence, the torque acting on the wheel shaft is measured and transformed via a calibration model to a viscosity of the fluid, they also found that the variation of the viscosity increases with increasing the water cut [23].

\section{Demulsification and demulsification techniques}

Demulsification is the process of breaking the emulsion into its individual incompatible phases, mainly water and oil. Demulsification process is very important process in petroleum industries, wherein, emulsions almost always occur either naturally or deliberately (man made emulsions). In petrochemical industries and petroleum refineries, separation of water from the crude oil is required before oil refining. currently chemical additives called emulsion breakers are massively used to break the water-in-oil emulsions. Technically speaking, the resistance of a w/o emulsions to coalescence and their response to the demulsification techniques such as thermal, mechanical, electrical or chemicals depends mainly on the Physico-chemical structure of the oil from which they are formed, emulsification conditions, and Aging. This means, the effort and Strategies for optimizing the w/o emulsion demulsification may vary from one oil field to another [1]. Thus far, various methods for breaking emulsions were introduced. These including, Chemical, Thermal, centrifugal, freeze/thaw, and electric demulsifications. chemical demulsification is the most widely used in oil fields $[3,24]$. In chemical demulsification, massive amount of chemicals are used to separate the water from the crude oils. However, most of these chemicals are expensive, toxic and un-ecofriendly. Therefore, other alternatives and sustainable demulsification methods are still in demends. In the folowing sections, various literature servey on the existing and potential future demulsification techniques is discussed.

\section{Chemical demulsification}

Chemical demulsification is one of the very crucial techniques of resolving water-in-oil emulsions, and it is massively applied in the petroleume industries. Basically, massive amount of surfactants can be prepared by just manipulating the existing surfactants, one way is by changing the acceptor, composition, quantity, and sequence of hydrophobic and hydrophilic groups in the commercial long chain polymeric surfactants [25]. The very basic fundamental of chemical demulsification mechanism of what so ever type of emulsion is that, the demulsifiers gradually replace the emulsifiers within the water-oil interfacial film, and that would eventually cause tremendous changes on the interfacial viscosity and elasticity [26]

Hafiz et al. had synthesized some novel demulsifiers for treating water-in-crude oil emulsions, they prepared cationic polymer of of diethanol amine easters by condensation and polymerization of the diethanolamine to diethanol amine polymers esterification reaction was carried out at temperature range of $140-160^{\circ} \mathrm{C}$ using different types of catalysts. Their demulsifiers were found to be effective in treating 
emulsions of refinery wastewater. The purity of the treated water was determined by turbidity measurement [27]. Alejandro et al. have altered structure of some commercially available demulsifiers (Alkylphenol polyalkoxylated resin and polyurethanes). The efficiency of the newly formulated products were assessed by several techniques such as: bottle test, rheometry, equilibrium interfacial tension, and transient changes in drop sizes distribution that was measured using Nuclear Magnetic Resonance. Emulsifiers were tested on experimental emulsion that was prepared by simply dispersing the brine in the oil without emulsifiers. that means, the asphaltene and resins originally existing in the crude oil did the job of the emulsifiers. Their results showed that, the best and the highest separations were observed when emulsions were injected with resin having intermediate polyoxyethylene and polyoxypropylene moieties [28]. Jiangying $\mathrm{Wu}$ and others have investigated the property and performance of 20 blocked copolymer from four different surfactant families on breaking model emulsions. Model emulsions were prepared by first diluting bitumen (5\%) in toluene, and centrifuging for five minutes to remove suspended indigenous small particles. This mixture was mixed with water and homogenised. polytron homogenizer was applied at $20000 \mathrm{rpm}$ for of three minutes. Their emulsion was observed to be stable without water resolution for three consecutive weeks. This emulsion was then broken via the aforementioned demulsifiers. Their resulted revealed that, the sequential block copolymer with more than $40 \%$ ethyleneoxide percentage was more effective than the others [25]. Wanli Kang et al. have studied the effect of several demulsifiers, namely, Phenolformaldehyde resin polyoxyethylene, polyoxypropylene and polyoxyethylene polyoxypropylene polymers. Their model emulsion was prepared by mixing purified kerosene and dewatered crude oil in a ratio of 7:3, then water was added drop wise. The demulsification process was carried out at $45^{\circ} \mathrm{C}$. Demulsification process was monitored in two distict samples, one without demulsifiers and other with demulsifiers. Results showed that, the strength and life time of the interfacial film was decreased when demulsifiers were added. Hence, the curve of the interfacial elasticity was found to decrease with increasing the demulsifiers' concentration, but came to a plateau of constant values beyond the critical concentration of demulsifiers. Masatto et al. have used Shirasu-porous-glass membrane to break water-in-crude oil emulsion, shirasu is a type of ash that is very rich in metal oxide mainly Silicone dioxide, however their results showed that Shirasu-porous-glass membrane could efficiently break water-in-oil emulsions with droplet sizes bigger than the average membrane pore diameter [24]. Delphine et al. have studied the effectiveness of nontoxic silicone based demulsifiers of polysiloxane copolymers on splitting water-in-crude oil emulsions. The test was carried out on experimental w/o emulsions, their emulsions which composed of $30 \%$ water were prepared at room temperature. polytrone homogenizer was used for mixing. To breack the emulsions, $2 \%(\mathrm{w} / \mathrm{w})$ of demulsifiers (polyoxyethylene-silicone triblock copolymers) were first dissolved in Xylene/methanol solution $(75 / 25(\mathrm{w} / \mathrm{w})$ before injecting them into the emulsions [3]. Similar experimental work also was reported by dalmazone et al. who investigated the efficiency of various formulations of these aforementioned non-toxic silicone based surfactants. The efficiency of the different formultions was determined by visual observation and measurement of water separation from emulsion, and dynamic interfacial tension measurement. They have tested silicone based formulation on two types of crude oils originated from North Sea and French oil fields. Their emulsification and demulsification methods were same as mentioned earlier [3]. Their results revealed that, poly siloxane demulsifirs were very efficient in breaking water-in-crude oil emulsions in comparison to some commercial demulsifiers.
Furthermore, some blend of silicone-silicone and silicone-organic was selected for their sustainability and versatility reason. Althogh silicone demulsifiers are claimed non-toxic and good potential alternatives, yet they are not lucrative to be commercialized for economic and cost reasons. However, the current increase in ecological constrains may favor them to be used in the near future or to be integrated in the formulation of the demulsifiers [29]. Svetlana et al. have investigated the effects of modified Chitosan derivatives in resolving o/w type emulsions. The study focused on the effectivness of hydrophobically modified (HM) Chitosan derivative demulsifiers copared to the commercial cationic polyacrylamide flocculant, and Unmodified (UM) Chitosan. The molecular weight of the demulsifiers was also modified. To test the demusifiers, experimental emulsions were made in laboratory. Sodium Dodecyl sulphate (SDDS) was selected as emulsifiers, emulsion was mixed for 10 minutes using ultrasonic mixture. was used to provide the mixing energy for an emulsification duration of 10 minutes. Results showed that, hydrophobically modified (HM) Chitosan derivatives with various molecular weights had shown to cause great separation compared to the others [30]. Abolfazl et al. have used the microfiltration membrane technology in separating the emulsion. They have tested the efficiency of the hydrophobic Polytetrafluoroethylene (PTFE) membrane having pore sizes of 0.45 micron meter in separating w/o emulsions. The experimental water-inoil emulsion was prepared in laboratory using span 80 (oil soluble surfactant) as emulsifying agent. Emulsions were prepared according to the agent in oil method, in which Span 80 was first dissolved in the crude oil then water was added gradually as drops, while agitation is going on. The water addition rate was $25 \mathrm{ml} / \mathrm{min}$. The mixing was carried out using pitched curve blade blender having 6 fins. Their results revealed that, the permeate flux from the membrane was found to be rich in emulsifiers and poor in water. Furthermore, the permeate flux was found to decrease with increasing pressure. The effect of the temperature was indeed obvious [31]. Ing Harald Auflem and others have studied the effect of pressure on separating w/o emulsions from North Sea crude oils. They prepared their emulsion by first mixing the emulsifiers (Silverson LURT emulsifiers) with the continuous phase (North Sea crude oil) then the aqueous phase was added gradually (Agent in oil method). A mixing speed of $2000 \mathrm{rpm}$ was applied for one minute (emulsification time). The demulsification process was carried out by introducing the aforementioned emulsion into a high pressure demulsification rig. Their results showed that the emulsion stability was to some extent related to the demulsification pressure. This stability-pressure relation is imparted to the fact that, when the rig pressure was reduced beyond the bubble point pressure, the low molecular weight portion of the crude oil could evaporate and form gas bubbles that evaporate from within the sample to the top part of the system. This massive movement of the bubbles and density differrences would put the heavy components in a floatation state. This floatation effects would induce the emulsifiers residing at the water-oil interface to dissipate in the gas phase (the bubbles). This dissipation would eventually render the droplets free from their encapsulating emulsifiers matrixs, hence, the neighboring droplets would merge together (coaleasce) and eventually separate into two phases (water at the bottom and oil at the top). another important finding of their investigation was that, by diluting the emulsion with toluene, the stability was also decreased and that is because dilution also decreases the viscosity as well as the density of the emulsion and also cause asphaltene to aggregate [32]. Nael Zaki et al. have used the carbone dioxide to break the w/o emulsions of various actual, as well as experimental emulsions. The dense $\mathrm{CO}_{2}$ was assumed to induce the Asphaltene fluctuation, flocculation and precipitation. Thus, the 
absence of asphaltene the strength of the interfacial film by causing the film thinning and film rupture and eventually droplets collision and phase separation. The experimental emulsion samples used in their study was prepared by mixing the two phases at $1500 \mathrm{rpm}$ using via Ultra-high speed Virtishear cyclone IQ Homogenizer for 2 minutes. emulsion stability was tested by visually opserving the water separation from the emulsion within duration of 24 hour. Then only the stable emulsions were chosen for demulsification via the aforementioned $\mathrm{CO}_{2}$ injection. Their results proved the possibility of using $\mathrm{CO}_{2}$ to treat water-in-crude emulsions of different characteristics and compositions [4]. Zhiaing et al. have investigated the demulsification effects of Poly (ethylene oxide (PEO), Poly (propylene oxide (PPO). They varied the molecular weight by altering the composition. Thus, $\mathrm{PPO} / \mathrm{PEO}$ ratios were varied through the anion polymerization process. the effectiveness of these modified copolymers on demulsification of the water-in-crude oil emulsions were tested on experimental emulsion coposed of $50-50 \%$ (w/o). The experimental emulsion was prepared in laboratory by mixing the aqueous phase with petroleum oil taken from Shengli oil field (China). Emulsion was mixed at $1200 \mathrm{rpm}$ for 5 minutes by HT-2 Homogenizer. For the demulsification process, Temperature and concentration of demulsifiers was fixed at $50^{\circ} \mathrm{C}$, and $100 \mathrm{mg} / \mathrm{L}$ respectively. The separation efficiency of the demulsifiers was determined by recording the water resolution for a settling period of 3 hrs. Result showed that, demulsification process can be enhanced with the reduction of the PEO fraction in the surfactant formulation [33].

Abdurahman et al. had made a comprehensive study on waterin-crude oil stability as well as demulsification. The investigated various stability parameters including, crude oil types, demulsifiers concentration, mixing speed, water volume fraction, and temperature. crude oils used in their study were originated from Iran and Malaysia. Several experimental emulsions were prepared according to the agent in oil technique, in which the crude oil is normally mixed with the emulsifiers first then water would be added gradually in drop wise fashion while mixing process goes on. The mixing energy was provided by three blade propeller, mixing time was fixed at 10 minutes. It was found that, the Iranian crude oil can produce more stable emulsion than the Malaysian Miri light crude oil [34]. Same authors, have studied the effect of the different types of the commercially available demulsifiers in splitting the aforementioned stable w/o emulsions. they have tested four different groups of demulsifiers, namely, Amine group, Polyhydric alcohol group, Sulphonate group, and polymeric group. beside that, the effects of the alcohol in the performance of demulsifiers also investigated [34]. Their results shwoed that, amine group demulsifiers were more effective in breaking the emulsion, followed by Acid then polymeric demulsifiers. From within the amine group demulsifiers, Hexylamine and diacylamine were the most effective commercial demulsifiers based on their their study [34].

\section{Electrical demulsification}

Electrical demulsification is a process of breaking of either waterin-oil, or oil-in-water emulsions by means of electrodes. Electrical demulsification devise is normally consists of two electrodes placed in opposed direction to each other. Emulsion is placed between the two electrodes. When, DC voltage is introduced to the system, electric field voltage is generated within the emulsion residing between the electrodes, that because the water in the emulsion is conductive. This will break the energy barrier between droplets, and water molecules shall immediately be hydrolyzed into hydrogen and oxygen gases molecules. The hydrolysis reaction is normally indicated through by hydrogen bubbles that evolve from the Positive electrode (cathode), and oxygen bubbles that evolve from the negatively charged electrode (anode). It was observed that, when the anode materials are made of metals with less oxidation energy than the system, these materials would dissolve in the system to produce metal ions instead of the evolving oxygen bubbles. Then these metal ions would react with hydroxyl ions that produced during hydrogen generation, to give metal hydroxides.

An electrochemical reactor consists of tank with two sets of metal electrodes (anodes and cathodes). These two electrodes are normally placed at certain specific distance apart from one another and both of which should be immersed in the system under treatment for oil contamination. The cathodes and anodes are mounted on the negative and positive outlets of a dc power supply of the reactor, respectively. Hence During the operation (emulsion treatment), the decaying metal ions are hydrolysed, such as $\mathrm{Fe}$ (II) (ferrous) and $\mathrm{Fe}$ (III) ions in the case of Iron metal cathodes [35]. For safety reasons, the electrodes are normally insulated from the emulsion to prevent short circuit [36]. A lot of parameters were reported to be affecting the performance and efficiency of the process. Harpur et al. have studied the ffect of $50 \mathrm{~Hz}$ sonosoidal electric field to treat and remove water from w/o emulsion. they used a horizontal rectangular container (electrostatic coalescer). Seven electrode modules were used, each model was $50 \mathrm{~cm}$ in length, and generating an electric field of $25-60 \mathrm{KV} / \mathrm{cm}$. Their results showed that the application of the electric can cause a significant growth in drop sizes. However, increasing temperature was found to accelerate the aggregation of the droplets [36]. Woo-in Jang et al. have also studied various parameter that affect the electrical demulsification techniques, Including, types of the electrode materials, polarization of the reactor, electrode size, temperature, oil concentration in the emulsion, mixing rpm, $\mathrm{pH}$, and electric power consumption.

The oil sample used in their study was waste of metal cutting oil, from Korea Houghton Corporation. The oil consisted of $80 \%$ mineral oil and 20\% surfactants plus some other chemicals such as anti foaming, bactericide, and anti corrosion. Experimental emulsion of the oil was prepared. The emulsion sample was introduced to the electrostatic coalescer and treated. Sample of the treated emulsion was taken from the reactor and analyzed after 30 minutes. Results showed that, aluminum reactor was performed better than the iron reactor, because, it gave high separation efficiency, and consumed less energy. Furthermore, water removal removal rate and energy consumption increased with increasing the applied potential. However, maximum separation of $94.05 \%$ was acheived at 60 volte, whereas, $85.50 \%$ separation was acheived at 30 Volts. Indeed, a wider electrode gave higher water removal and less energy consumption compared to narrower electrode, beside that, the best operating temperature was found to be $50^{\circ} \mathrm{C}$, and variation of the oil volume fractions were found to have no effects on the demulsification process. Furthermore, agitation found to reduce the separation rate, while low $\mathrm{pH}$ was observed to be more effective than high $\mathrm{pH}$. However, when the treated sample was analyzed, it was observed that there is a gradual increase in the particle sizes with time, hence their general conclusion had conformed the ability of the electrocoalescence process to treat the industrial oil-in-water emulsion [37]. Gary Sams and Zaouk have investigated all the upstream production parameters of petroleum wells, and their effects on emulsion separation. results revealed that, more vigorous and powerful electrostatic techniques are needed to treat and resolve the tight emulsions [38]. Simone less and others have used a device named Aibel vessel electrostatic coalescer, to break petroleum emulsions. The separation efficiency of the devise was evaluated in terms of percentage water resolution, and droplets sizes distribution, before and after the treatment. However, their results 
showed that, this system can effectively reduce the water content in the emulsion. But, chemical addition was found to greatly accelerate the separation process. Tsunki Ichikawa and others have applied electric field to break oil-in-water emulsion. Their experimental emulsion of various volume fraction was prepared by simple hand shaking, around 100 times. Their electrostatic device was consisted of two stainless steel plates fixed in opposite position inside glass container. Emulsion sample was placed in the device and the external electric field is applied. Their results revealed that, higher concentration of the electric had given high separation rate, but increasing the amount of ionic surfactants does not increase the separation rate. Although, Their devise was very good in breaking oil-in-water emulsions, yet it could not break the w/o emulsion even at elevated electric field, and frequency as high as $10 \mathrm{KHz}$ [39]. Harld Fordedal and co-worker have studied the percolation behavior of water-in-oil emulsions under the influence of the electrical field. Percolation refers to the abrupt increase in dielectric constant of the emulsion, when its volume fraction attains a maximum values at constant temperature. They have prepared two experimental emulsions for this reason, one of which is stabilized by the naturally occurring surfactant (Asphaltene and resin), and the other is stabilized by the commercial surfactants. Their results proved that the dielectric constant of the emulsion was found to increase with the increase of strength of the electric field; this was observed with the water-in-oil emulsion stabilized with the indigenous interfacial active surfactants, wherea, the reverse effect was observed with the emulsion stabilized by the commercial surfactant. Thus, they concluded that, the dielectric properties of the commercial surfactants are by far different than that of the indigenous surfactant (Asphaltene and resins) [40]. Junji et al. have applied the electrostatic techniques to break water-in-oil emulsion hence they used some kind of metal oxide chemicals that are assumed to induce the increase in the coalescence rate of the crude oil droplet under the influence of the electric field, thus the performance of the device was assessed via video microscop from which a digital picture was captured and transferred to a computer for further analysis, their study revealed that, the shapes of the droplets were observed to change from spherical shapes to hemispheres when adsorbed at the electrode surface also the metal ions used were observed to be oxidized indeed the ion concentrations of the metal ions was observed to increase at the oil-water interface, also the image analysis of the coalescence of the neighboring droplets was observed to depend on the electrical potential applied [41]. Bailes et al. have combined the air bubbling process with electrostatic demulsification. However, their results concluded that, bubbling had enhanced the separation efficiency. Indeed, separation rate also was observed to increase with increasing the air flow rate [42]. Tsuneki Ichikawa et al. have used a low electrical field to destabilize oil-in-water emulsion, hence the low electric field was assumed the reduce the thickness, and weaken the strength of the repulsive double layer, due to movement of the ions [43]. Seiji Kanazawa et al. have developed an apparatus for electrostatic coalescence using the electrostatic atomization techniques. The system was tested have tested on two types of emulsions. One was composed of water droplets dispersed in silicone oil, and the other was a stable emulsion that was prepared without using emulsifiers. The test was excuted by monitoring the movement of the positively charged oil droplets as a response to the applied electric field. thus they have first observed the marked drops in the transparent oil, then after the DC electric field was applied, the individual droplets were observed to aggregate and their traveling distance from the electrode, and the traveling energy were monitored [44]. Others have investigated the electrodynamic mechanism of the electrostatic demulsification process, via simulating the effect of the field strength on an individual water droplet [45]. Yakhkeshi et al. have used a uniform electric field to treat oil-in-water emulsion. They aimed to observe how the electric current could induce the scattered find droplets within the emulsion to aggregate and come close to one another, and produce bigger sized droplets. Their emulsion was consisted of benzene as the oil phase (model oil) and water. Emulsion was mixed by a magnetic stirrer for 15 minutes. (emulsification time). SDDS was used as emulsifying agent in a dosage range of 2-4 ppm. The dispersed Phase volume fraction was $4 \%$ of total emulsion volume. Their result again consolidated the usefulness of the electrostatic demulsification process on breaking the oil-in-water emulsion. The influencial parameters were, type of the insulator at the surface of the electrode, types of the oils, oil volume fraction in the emulsion, temperature and the electrical voltage [46]. Mir et al. have used nonuniform electrostatic techniques to treat simulated waste water emulsions. They formulated the experimental waste emulsion by mixing Xelene, water and metals, and surfactants. The volume fractions of the oil ans surfactants were $2 \%$ and $1 \%$ respectively. Ultrasonic mixture was applied for 6 minutes. For demulsification process, The electrostatic apparatus consisted of two electrodes that mounted oppositely in glass chamber. Three types of the electrode metals, namely Iron, Tungsten and copper were tested. However, their results proved that, Copper electrode was the best and performed better than the others. Indeed, beside the the type of electrode, voltage, temperature, and time were also crucial parameters. The highest separation was observed at electric power of $5400 \mathrm{~V}$ and temperature of $45^{\circ} \mathrm{C}$. Akuma Oji et al. have applied the High Voltage Dielectric Current (HVDC) having voltage range of 4- $16 \mathrm{KV}$ to break real oil field emulsions from Obagi oil field. They investigated the effects of variating the distance between electrodes made up of Zinc. The distance was altered gradually from 60 to $244 \mathrm{~mm}$. The operation time also ranged from 10-40 minutes. The best operation condition was observed to be $8 \mathrm{KV}$ voltage and 122spacing distance [47].

Byoung et al. have used a high AC field dehydrator to treat waterin-oil emulsions. They used 2-20 KV Voltage, and frequency range of $60-2000 \mathrm{~Hz}$ was applied [48]. They studied several parameters, including chemical demulsifiers, operating temperature, operating time and residence or contact time in terms of the feed flow rate. Their experimental model w/o emulsion of $20 \%(\mathrm{v} / \mathrm{v})$ water contain was used via electromagnetic stirrer (Heidolph RZR 205, Germany), at $1000 \mathrm{rpm}$ and 10 minutes emulsification time. The electrostatic hehydrator device was made up of glass chamber, and the electrodes that were insulated with glass material to prevent possible short circuiting. Prior to the demulsification, certain amount of chemical demulsifiers were added to the emulsion and mixed for 5 minutes at $1000 \mathrm{rpm}$, then transferred into water bath to attain the desired temperature before transferring to the electrostatic coalescer. the flow rate was monitored to give a residence time of three minutes. Their results showed that, separation efficiency increased with increasing the electric field. also adding the demulsifiers was found the increase the separation efficiency furthers to $80 \%$, while the temperature and the residence time were not that effective [48].

\section{Thermal demulsification of emulsions}

Thermal treatment is referred to the use of temperature to break petroleum emulsions. Conventional hot plate is used in lab scales to provide the optimal temperature. Beside that some researchers had treated the emulsion by reducing their temperature up to beyond freezing point then rising the temperature gradually, this is known as the freeze/thaw method. However, in most cases, thermal treatment is applied jointly with chemicals to improve the efficiency, since 
temperature can tremendousely reduce viscosity of emulsions.A group of researchers have reported the usefulness of the thermal demulsification techniques in breaking crude oil emulsions, fore instance Abduraman et al. have investigated the use of hot-plate in breaking w/o emulsions in comparison to the microwave demulsification techniques. The test was carried out in an experimentally prepared emulsion sample of two types of pure crude oils that were donated by PETRONAS (A Malayisan based oil and gas company). The experimental emulsion was prepared in $500 \mathrm{ml}$ beaker with different volume fraction of oil and water phases. Mixing energy was supplied by standard three blade propeller at $1600 \mathrm{rpm}$, for 5 minutes at ambient temperature of $28^{\circ} \mathrm{C}$. the volume fraction of the water in the emulsion was varied as 30, 40 and $50 \%(\mathrm{~V} / \mathrm{V})$. Befor demulsification, emulsions were tested for stability and quality, and only stable emulsion were considered for treatement. Their results revealed that, microwave have shown great separation efficiency over the conventional hotplate heating, and that is because of the heating patterns. since microwave heat materials volumetrically according to their dielectric properties. Thus, its energy is generated within the molecules of the materials unlike the conventional hotplate, in which heat is transferred from the surface of the hotplate to the bottom surface of the sample holder, then to the surface of the sample, then to the bulk of the sample. This makes the conventional heating slow and non-uniform. Another thing is that, microwaves are electromagnetic waves, so they have high potential to neutralize the electromagnetic repulsive barrier (Zeeta potential) between the droplets, this together with reduced viscosity as temperature increase, would ease the droplets aggregation and coalescence [34]. Raman Morales Cherbrand et al. have tested the combination of three distinct techniques, namely, Heating, Enzymatic and centrifugation to break emulsion that is originated from soybean oil. Soybean oil was extracted by dispersing the soybean powder in $2 \mathrm{~L}$ of aqueous water phase ( water comes in contact with oil) at $200 \mathrm{rpm}$. the $\mathrm{pH}$ was increased to 8 by adding certain amount of sodium hydroxide. the stirring process had continued to 15 minutes, after which three distinct phase were separated by centrifugation. Befor centrifugation, soybean cream was initially mixed with water to adjust its $\mathrm{pH}$, then $1 \% \mathrm{w} / \mathrm{w}$ of the enzyme was added then shaked at $15 \mathrm{rpm}$ and $50^{\circ} \mathrm{C}$ for three hours. After that Enzyme were deactivated by heating at $95^{\circ} \mathrm{C}$. After that the sample was centrifuged to separate the oil. In another step, the enzymetic treated sample was heated at $95^{\circ} \mathrm{C}$ for $30 \mathrm{~min}$, then cooled in a chilled water bath and centrifuged. In a third step, a fresh set of enzyme-treated cream held in a freezer at $-18^{\circ} \mathrm{C}$ for $24 \mathrm{~h}$, then thawed at $30^{\circ} \mathrm{C}$ for $3 \mathrm{~h}$ prior to centrifugation (Ramon Morales). Tov et al. have studied the effect of heat and chemical demulsifiers on w/o emulsion resolution. they investigated the effect conbing the two techniques instead of any of them alone. Experimented oil spill emulsion was formulated for testing by rotating flask methods. Their study had led to the following conclusions, w/o emulsions of paraffinic petroleum could break faster than that of the high asphaltene oil. Heat treatment could break the emulsion slowly but after adding chemicals the separation rate had increased. indeed for viscous emulsion it had better to introduce the chemical demulsifiers at $10^{\circ} \mathrm{C}$, whereas, for highly viscous emulsion, it had better to add the demulsifiers after heating the emulsion. Moreover, emulsion formed from the distillation residue could be broken with moderate heating, while that with diesel oil were not broken even at high temperature. also as emulsion breaks down, its viscosity reduces [49]. Chantal et al. have used the insitu emulsion burning techniques to treat the oil spill emulsions. Their intension was to minimize the oil spill pollution. They investigated certain specific parameters that include the mechanism of the burning process, the techniques used for ignition, the environmental disturbance that burning could cause, the burning process was excuted on iced medium.Their results had drawn so many ideas and findings. Fore instance, they identified the characteristics of the specific igniter, that should be used to ignite the crude emulsion burning. Thus, Helitourch was identified as one of the most effective ignition techniques for crude oil emulsion burning. Beside that the basic requirement for any igniter for this purpose must be composed of lightly fueled front part, and heavily fueled back part, and must be provided with quick sparking to start the ignition. Indeed, anti-foaming additives are required to improve the flame spreading capability. The burning efficiency acheived by this method, had reached up to $75 \%$. The burning residue consisted of heavily viscous and tar-like component with some minor raw emulsion [50]. Bernard et al. have studied effect of temperature on separating w/o emulsion that stabilized by wax particles. Experimental emulsion was prepared by first dissolving the wax particles in squalane. Emulsion types was checked by drop test, and conductivity measurement. Stability test was performed by testing the amount of water or oil resolved from the emulsion within a fixed period of time. Temperature increased the extend of coalescence, that is because at elevated temperature, the wax particles at the interface would fuse, melt vanishes from frfrom the interface, and this would induce the droplets to easily come together and merge (means separation). The barrier collapsing temperature is normally the same as the melting temperature of the pure wax particles alone. However, if emulsions were prepared at emulsification temperature that is close to wax melting temperature, then it was observed to be stable even at elevated temperature [51]. Taylor et al. have studied the effect of temperature on breaking bitumen emulsions that is originated from Wolf-lake (Canada). the experimental emulsion was stabilized by ethoxylated nonylphenol surfactant.Their results showed that, as the temperature of the system getting closer to the cloud point, emulsion could break gradually. Indeed, the bitumenwater interfacial tension was found to decrease with increasing temperature, and found to be very small at cloud point temperature [52]. Basically, the mechanism behind the effect of temperature on emulsion separation is that, at low temperature below the cloud point, the system was observed to poses micelles plus free surfactants that would stabilize the bitumen-water interface, but at elevated temperature the ethoxylated groups could be dehydrated, and consequently the hydrophilicity of the surfactants could be reduced, which means their tendency to dissolve in the continuous water phase would reduce, and eventually would causes the phase separation. although at high temperature, ethoxylate group have the tendency to dissolve in the oil phase, but in their study, they mentioned that the complex structure of the bitumen will hinder the ethoxylate group to dissolve in the bitumen (oil phae), so they would aggregate as separate phase letting the two phases to separate into pure bitumen and water [52]. Guohua and Gaohong have studied the freeze/thaw method, which involves the cooling of the sample to a very low temperature (below the freezing temperature -40 for exemple), then increase the temperature backward gradually. However, their emulsion was real emulsion taken from the plan, Small amount of sample was placed in a centrifuge bottle, and cooled to freezing temperature, then thawed back to certain temperature, and then centrifuged for $5 \mathrm{~min}$ at $400 \mathrm{rpm}$. The volume of the water separated from the emulsion was read off through scales on the centrifuge tube (sample holder). They found that, demulsification of the water-in-crude oil emulsion depends strongly on certain parameters including, the original water contents, freezing temperature, Freezing period as well as thawing speed and temperature. The optimal freezing temperature in their results was found to be around $-40^{\circ} \mathrm{C}$, for the oil sludge taken from the used oil. indeed high initial water volume 
fraction and milder thawing would lead to increase in the volume of water separation. Beside that, the optimal thawing conditions were found to be either Air at its ambient temperature, or water bath at temperature below $20^{\circ} \mathrm{C}$. and the separated water was found to contain some organic contaminent, during the freeze/thawing process, the formation of the surfactant micelles also was observed. theses micelles were greatly affected the quality of the separation [53]. Also Chang Lin et al. have used the freeze/thaw method to break water-in-oil emulsion that produced from emulsion liquid membrane. However, their experimental emulsion was produced using the agent in oil method, wherein, the surfactant (Span 80) was dissolved in the oil phase, then the de-ionized water was dispersed while the mixing was going on at a mixing speed of $1000 \mathrm{rpm}$ to coursen the system first, then the mixing speed was increased to 2000 and eventually to $6000 \mathrm{rpm}$ for 20 minutes, to get the submicron sized droplets emulsions. and from their results they have revealed that the freeze/thaw method was very effective and easy to handle process to separate w/o emulsions. and the mechanism behind the freeze/thaw method follow the collision theory, in which drops are moved close to each other gradually when the mechanical barrier capturing them is gradually removed. The fundamental mechanism behind the freeze/thaw demulsification method is that, during the transformation of the water phase from liquid to ice the volume expansion of the droplet could take place, and that would cause partial or full coalescence. Hence, these coalesced small droplets could diffuse into the big droplets, because of the interfacial area shrinking that occurs during the heating (thawing) process. Again, the most effective parameters in freez/thawing process, were the amount of the water content in the emulsion. Also, freezing with dry ice was found to be more effective than that with the refrigerator. but preferentially the optimal freezing temperature should be below the solidification temperature of the droplets, to achieve a suitable and uniform crystallization [54].

Xiaogang yang et al. have studied the stability pattern of asphaltene stabilized emulsions, and the effect of the Freeze/thawing on the demulsification process. the parameters of concern were the surface tension, Zeeta potential, the water droplets and the water separation from emulsion. Their experimental emulsion was prepared by choosing the dodecane as a model oil and deionized water as an aqueous phase. the indigenous surfactants (Asphaltene and resin) used in their study were extracted from crude oil via the n-alkane precipitation method, then the experimental emulsion which contain $30 \%$ water was prepared by mixing the dodecane with the deionised water and emulsifiers (Asphaltene and resins) [55]. The concentration of the Asphaltene was varied in the range of 3 to $9 \mathrm{~g} / \mathrm{l}$, and that of Resin was varied in a range of 0.47 to $3.3 \mathrm{~g} / \mathrm{l}$. The system was mixed 300 time by simple hand shaking method. Their results revealed that, emulsion stability is very strong function of Asphaltene and Resin concentration, because their adsoption at the oil-water interface would produce a film that prevent coalescence. However, their surface activities were observed to decay with increasing asphaltene concentration. Also Zeeta potential of the asphaltene and resin stabilized emulsions was observed to be negative, and increases with asphaltene and resin concentrations. for demulsification by thawing process, the separation was more easy in resin stabilized emulsions than their asphaltene stabilized counterparts. Further more, the air thawing was found to remove more water than the water thawing, indeed, the thawing with microwave heating was found to be very effective than thawing with air and water [55].

\section{Other Demulsification Methods}

This section would consider mostly the mechanical methods that are in common use for treaiting crude oil emulsions. That including, centrifugal, membrane and so on. Habn et al. have investigated the effects of ultracentrifugal demulsification on breaking w/o emulsions. the emulsions were made in laboratory by the use of several emulsifiers such as Gantrezan 119, Tween 80, and sponto 221, each with various concentration ranged between $0.05-0.01 \%$. The oil volume fraction in the emulsion was fixed at $30 \%$, the mixing energy was supplied by Waring Blender for a mixing period of 30s. For the demulsification process, the centrifuge efficiency was investigated by direct measurement of the droplet sizes before and after the processing [56]. Kashmiri et al. have investigated the effect of ultracentrifugal method on demulsifying $\mathrm{o} / \mathrm{w}$ emulsions. They produced several experimental emulsions with different concentrations of the emulsifying agent, namely, Tween 20 , Triton $\mathrm{x}-100$. The oil to water volume fractions were fixed at $50-50 \%$. The demulsificatiom process was carried out by high speed ultracentrifuge, which was operated at the speed of $36,460 \mathrm{rpm}$, at ambient temperature of $25^{\circ} \mathrm{C}$. However, result showed that the rate of separation was found to be independent of emulsifiers' concentrations [57]. Beside that Abdurraman et al. have investigated the feasibility of using the high speed centrifuge in separating the coconut milk emulsion into pure water and coconut oil. the parameters of interest were the speed of the centrifuge (rpm), which was in the range of 6000 to 12000 , and centrifugation time that was ranged between 30 to $105 \mathrm{~min}$. Their results revealed that, among the four centrifugational speeds (6000, 8000,10000 , and 12000) used, only $12000 \mathrm{rpm}$ had given the optimal separation efficiency. Centrifuge separate emulsions in accordance with the general centrifugation theory, which tells us that, the suspended particles always move in the opposite direction of the centrifugal force, and separation occurs because of the density difference. The processing time was also segmented into 6 different parts, 30, 45, 60, 75, 90, and 105 minutes. Temperature was fixed at $30^{\circ} \mathrm{C}$, and there again the best separation efficiency was observed with the centrifugation time of 105 min [34]. Indeed Kocherginsky et al. have used the microfiltration membrane to break w/o emulsion. The fundamental theory behind that was the oil droplets would fluctuate at the membrane pore. Factors of concern were, types of membrane, membrane pore size, the transmembrane pressure, membrane thickness and the initial water concentration. Their liquid membrane emulsion was prepared by mixing kerosin and alkali aqueous solution and di-2-ethylhexyl phosphoric acid then the mixture was agitated vigorously at $1000 \mathrm{rpm}$ via mechanical mixture for an emulsification period of $1 \mathrm{~h}$, at ambient temperature. Their results revealed some interesting fact including the possibility of using the polymer membrane as a good choice to break w/o emulsion. though some parameters such as membrane material, Pore sizes, and transmembrane pressure, had very major effects on the separation process. also another important observation was that, not all the membrane types are suitable for treating w/o emulsions. Hence, according to their results, hydrophilic membrane is the only one that could break the w/o emulsions with pore sizes smaller than the droplet sizes indeed increasing the transmembrane pressure also was found to increase the separation efficiency, but membrane thickness was found to be not effective in the separation efficiency, hence the separation process is based on the interaction between the droplets and membrane not based on the sieving effects [58]. Nalin Nadarajah et al. have used some sorts of bacterial culture to break water-in-petroleum oil emulsions. They have collected the bacteria from crude oil contaminated soil. After the isolation, the bacteria is kept in cyclone fermenter on mineral salts, then various bacteria colonies were extracted and purified. A model (kerosene) emulsion was prepared by first dissolving the emulsifiers in the aqueous phase. For demulsification experiment, $1 \mathrm{ml}$ of the bacterial colonies was 
added to $9 \mathrm{~g}$ of model oil emulsion and mixed for $20 \mathrm{~s}$, and incubated at $50^{\circ} \mathrm{C}$ under static condition. The demulsification efficiency of the bacteria was determined by simply measuring the water separated from the bacteria treated emulsion, and compared with that of the control sample. From their results, they have observed that, it was difficult to prepare a model emulsion of the crude oil that mimic the actual crude oil emulsions because of the complexity found in the crude oil, the best separation efficiency for the biological demulsification was observed at $50^{\circ} \mathrm{C}$, and that was expected since the elevated temperature reduces the viscosity of the oil and emulsion, and increases the density difference between the two phases, and weaken the interfacial barrier which leads automatically to droplets coalescence. The culture age was found to be not important in the demulsification. However, culture cultivated from crude oil medium had given high demulsification efficiency than culture cultivated from motor oil and diesel [59].

Tereza Neuma et al. have applied the microemulsion system to break w/o emulsion of Brazilian crude oil. A moded microemulsion system was prepared in laboratory, the aqueous phase contained $5.2 \%$ HCL, the oil phase was toluene, and the emulsifying agent was isopropyl alcohol. The demulsification experiment was carried out by directly contacting the microemulsion and w/o emulsion in centrifuge tube for a duration of $30 \mathrm{~min}$, with continuous mixing and fixed temperature of $70^{\circ} \mathrm{C}$. The separation efficiency was calculated as the percentage of water separated with respect to the original amount of water used. From the results, microemulsion was proven to be a suitable method to break w/o emulsions formed during the mining process of the Brazilian oil. The original water content of the system had a great effect on the process [60]. Del Colle et al. have used the alumina tubes as a filter material to split water-in-sunflower oil emulsions using tangential filtration process. They used a ceramic tube that was made of alumina. The tube was first sintered at $1450^{\circ} \mathrm{C}$, then impregnated by zirconic material then calcinated and heat treated at temperature range of 600$900^{\circ} \mathrm{C}$ to discard the light organic materials, and convert the zirconium to zirconium oxide that was impregnated in the aluminia in the forms of nanoparticle aggregates. This new microporous membrane was tested in a microfiltration of aqueous system to assess its performance. The parameters investigated were, the transmembrane pressure, and input emulsion flow. Results showed that, the ziconia impregnated model was found to be very effective and could operate at very high temperature of $900^{\circ} \mathrm{C}$ [61].

Mohtada Sandrazadeh et al. have used the PTFE membrane to treat $\mathrm{o} / \mathrm{w}$ emulsion that was originated from wastewater. A simulated waste water model emulsion was prepared experimentally by mixing gas oil and distilled water. Emulsion was mixed using a pitched curved blade blender with 6 fins, for $10 \mathrm{~min}$. Their results showed that, increasing temperature, pressure, as well as flow rate would affect the permeate flux. No fouling was observed and the water content of the permeate was to increase with increasing the temperature and pressure and decrease with increasing the flow rate [62]. Aileen Lozan et al. have used salts to induce the flocculation of oil-in-water emulsion. that was stabilized by anionic emulsifiers Sodium dodecyl sulfate (SDDS). o/w nano emulsion was prepared experimentally by two distinct steps: in step one, the low energy emulsification method used, wherein no heat or vigorous mixing is required, only mixing of oil, water, surfactants, alcohol and appropriate amount of salt and aqueous phase to make a bicontinuous emulsion system. In the second step, that bicontinuous emulsion system was injected in large amount of water with slow mixing to produce nanoemulsion system of fixed drop sizes which was further diluted in sodium dodecyl sulfate solution to get the desired oil-in-water emulsion [63].

\section{Microwave demulsification}

Electromagnetic spectrum laying within $300 \mathrm{MHz}$ to $300 \mathrm{GHz}$ is known as microwave. Microwave have electric and magnetic properties. thus, when they are projected to materials they obey the optical rules of transmission, absorption and reflection depending upon the medium characteristics, hence, the applied field will induces a polarization effect to the medium. The wave length vary from $1 \mathrm{~mm}$ to $1 \mathrm{~m}$, in accordance with aforementioned frequency which is between $(300 \mathrm{MHz}$ to $300 \mathrm{GHz}$ ). Beside heating and scientific research purposes, some frequencies are reserved for specific purposes, including these of cellular phone, radar, and television satellite communication, by Federal Communication commission (FCC). However, the most frequently used frequency for Industrial, scientific and medical (ISM) purposes are 915 and $2450 \mathrm{MHz}$, with 915 is used in this research [64]. Microwave is commonly preferred in material processing over its conventional counter part, due to its volumetric heating. The mechanism of heating in conventional heating occurs through diffusion from the surface of the material to the bulk. whereas, in microwave heating, the temperature gradient in different location within the sample is almost invariant, another important phenomenon is that different material possess different heating pattern, and that is due to the variation in material's microwave absorption capacity which in turn depends on dielectric properties [64].

\section{Equation of heat transfer in microwave}

The general form of heat transfer equation consists of three principal forms which are conductive convective and irradiative form given bellow.

$$
g_{M W}=\frac{h A}{V}\left(T_{m}-T_{a}\right)+\frac{\varepsilon A \sigma}{V}\left[\begin{array}{c}
\left(T_{m}+273.15\right)^{4} \\
-\left(T_{a}+273.15\right)^{4}
\end{array}\right]+\rho C_{p}\left(\frac{d T}{d t}\right)
$$

Unless this is not the case in microwave heating, wherin heat is generated within the material and it is transfer property is directly linked to the thermophysical properties of material as reported by curet and others. the detailed heat transfer mechanisms in microwaves will be explained in the following equations.

$$
\rho c_{\mathrm{p}} \frac{\partial \mathrm{T}}{\partial \mathrm{t}}=\operatorname{div} \cdot(\mathrm{k} \nabla \mathrm{T})+\mathrm{Q}
$$

Where: $\rho$ and $C_{p}$ are density and heat capacity of sample being heated, $\frac{\partial \mathrm{T}}{\partial \mathrm{t}}$ rate of temperature increase and $\mathrm{Q}$ is the internal energy acquired by the sample from the dissipated microwave power and could be calculated for $\mathrm{Z}$ directed electromagnetic wave by an equation relating power drop from surface to bottom which is:

$$
Q_{z}=\frac{F_{0}}{d_{p}(z)} \exp \left(-\int_{0}^{z} \frac{d z}{d_{p}(z)}\right)
$$

$\mathrm{F}_{0}$ : Microwave surface flux and $\mathrm{d}_{\mathrm{p}}$ is the penetration depth which is strongly related to dielectric properties of the sample designated as the distance at which the microwave represents $1 / \mathrm{e}$ of the surface power flux and its mathematical expression is:

$$
\mathrm{d}_{\mathrm{p}}=\frac{\mathrm{c}_{0}}{2 \pi \mathrm{f}}\left[2 \varepsilon\left(\left(1+\left(\frac{\varepsilon^{\prime \prime}}{\varepsilon}\right)^{2}\right)^{\frac{1}{2}}-1\right)\right]^{-\frac{1}{2}}
$$


While surface flux at the same conditions as Maxwell equations is calculated by the following relation.

$$
\mathrm{F}_{0}(\mathrm{x}, 0)=\frac{2 \mathrm{P}_{\text {surf }}}{\mathrm{ab}} \sin ^{2}\left(\frac{\pi \mathrm{x}}{\mathrm{a}}\right)
$$

Where $(a, b)$ are the dimensions of the wave guide

However, based on Maxwell equation perspective, the magnitude of microwave power absorbed by the dielectric samples can be estimated by the local electric field strength on the sample being treated as

$$
\mathrm{Q}_{\mathrm{abs}}=\omega \cdot \mathrm{E}^{2} \cdot \varepsilon \cdot \varepsilon^{\prime \prime}
$$

The electric field for plane wave propagation mode is solved by the following equation

$$
\nabla \times\left(\mu_{\mathrm{r}}^{-1} \nabla \mathrm{E}\right)-\left(\dot{\varepsilon}_{\mathrm{r}}-\mathrm{j} \frac{\sigma}{\omega} \varepsilon_{0}\right) \mathrm{k}_{0}^{2} \mathrm{E}=0
$$

$\omega:$ Pulsation of microwave radiation $(\mathrm{Rad} / \mathrm{s})$

E: electric field intensity $(\mathrm{v} / \mathrm{m})$

$\varepsilon$ : Complex permittivity

$\sigma:$ electrical conductivity $(\mathrm{s} / \mathrm{m})$

$\mu \mathrm{r}$ : relative electromagnetic permittivity of material $(\mathrm{H} / \mathrm{m})$

$\mathrm{k}$ : thermal conductivity (w/m.K)

According to some previous researchers, in the above equation the radiative and convective terms are very minor and that is because sample container has very low dielectric constant therefore it does not generate much of heat and hence most of the waves are transmitted through the glass sample container to the emulsion. The density and heat capacity of emulsion are calculated from the following mixing rules:

$$
\begin{aligned}
& \rho_{m}=\rho_{w} \varphi+\rho_{o}(1-\varphi) \\
& C_{p, m}=C_{p, w} \varphi+C_{p, o}(1-\varphi)
\end{aligned}
$$

Where:

$$
\rho_{m}, \rho_{w}, \rho_{o} \text { : are the densities of emulsion, water, and oil respectively }
$$

$C_{p, m}, C_{p, w}, C_{p, o}$ : are the heat capacity of emulsion, water, and oil respectively.

\section{Some literatures on microwave demulsification}

Different materials have different heating capabilities when exposed to the incident microwave energy. Hence, in opaque materials, the waves are totally reflected, while in transparent materials, they are transverse, but in dielectric materials, waves are absorbed and transformed to heat. However, introducing some specific metal powder (Susceptive) to non conducting solvent would render them microwave active and they can be heated [65]. The treatment of crude emulsion is of major importance in the petroleum industries, since most of the world's crude oils are produced in the forms of emulsion. Water is normally a good microwave absorber, because of its high dielectric properties, however, in some repports, at large samples, the heat transfer in oil was faster than that in the water and that is imparted to the thickness of the oil, but this convention does not work with small sample hence for small samples, the heating rate of the water was faster [66].
Emulsion was first treated via microwave by Wolf who was first started the concept of microwave demulsification. Since then, more and more researches have been devoted to this field. The efficiency of microwave on emulsion was found to induce some effect on the treated emulsion such as, the increase in the temperature shall lead to reduction in viscosity, and this would increase the mobility of the water droplet, that may in turn neutralizes the zeta potential of the dispersed droplets, and also break the hydrogen bonding between the water and the surfactant molecules. Beside that, the electromagnetic wave is believed to increase the internal pressure of the water droplets, leading to reduction of the interfacial film thickness and charges surrounding water droplets, and that would set the water droplets free to move toward each other, and downward by gravitational force [67]. the advantage of applying microwave energy over its conventional counter part is the ability to heat sample more uniformly than the conventional heating, although in some cases local overheating may occurs causing Hotspot or thermal runaway within the sample. Crude oil consists of vast numbers of components that differ in their conductivity and polarity, and absorptive patterns with asphaltene being the dominant and the major charge carrying component $[68,69]$.

In another report the effects of container types and water to oil ratios on emulsion stability was studied, the types of containers used in that experiment were non reflective and partially reflective ceramicmetal complex plates with emulsion being placed in the open exposed part. The maximum power distribution (resonance $\mathrm{R}$ ) is determined by plotting the power calculated against various parameters, including dispersed phase volume fraction, emulsion sample thickness and plate types. The averages power absorption for $\mathrm{o} / \mathrm{w}$ emulsion was greatest in sample heated with reflective surface, and decrease with increasing oil volume fraction. The thermal runaway was estimated by plotting the difference between the maximum and minimum temperature of the samples (o/w emulsion), plotted versus irradiation time. It was found to increase with increasing dispersed phase volume fraction [69]. Another observation was that unlike the o/w emulsion, in w/o emulsion there was a decrease in the amplitude at metal plate-emulsion interface, this situation is claimed to arise because of small dielectric loss of the later case, beside that also it does not respond much to water volume fraction variation. Numerous investigations have been done in the field of microwave heating and material processing. Christian et al. have studied the absorption mechanism for emulsion containing aqueous and nano droplets, and found that absorption is attributed to the types of ions, their concentrations, as well as their polarization [70]. Others have studied the effect of microwave in treating industrial waste water emulsions, and admitted that microwave can be used as an alternative to the existing demulsification methods, however, addition of small amount of acid was reported to increase the efficiency, moreover, the effect of aqueous phase composition on microwave absorption was investigated by Montserrat et al., [71].

Bjerdalen et al. have applied the microwave and ultrasonic techniques to treat the solid particles deposited at the petroleum well. These particles consist mainly of asphaltene, resins, and precipitated wax, together with other indigenous solid particles, such as silica, bentonite and gypsium. The parameters of concern were, temperature, Viscosity, and concentration of crude oil. The microwave device used was sumsung Mini-Chef MW $101^{\circ} \mathrm{C}$ that had a frequency of 60 $\mathrm{Hz}$, and output power of $120 \mathrm{~V}$. The temperature measuring probe was of type Greenlee THH-500. While the ultrasonic device was Medson mysono 201 A19001213 that had power output of $15 \mathrm{~V}$ and frequency of 3.5 MHz. The crude sample was originated from SANTA BARBARA. The processing time for microwave, was 80 and 180 
seconds. Whereas, for ultrasinc treatment it was varied as $30,60,180$ seconds. Results proved that, microwave irradiation could elevate the temperature of petroleum crude oil and asphaltene and decrease the viscosity, while with ultrasonic treatment the viscosity was observed to increase with increasing the irradiation time [72]. Abdurahman et al. have investigated the separation of water from crude oil by sing conventional heating and microwave heating comparatively. the study was carried out on two different types of crude oils, and two different water to oil ratios of $30-70 \%$ and $50-50 \%$ w/o emulsions. Experimental emulsions were prepared by mixing the two phase vigorously via the standard three blade propeller, at mixing speed of $1800 \mathrm{rpm}$, in ambient temperature for $5 \mathrm{~min}$. Three types of emulsifiers, namely Low sulfur wax residue (LSWR), Triton-x100 and Span 83 were used to produce the most stable w/o emulsion. Elba domestic microwave oven was used for demulsification. The temperature profile of the sample was monitored using three thermocouples that were inserted in the sample at different location (Top, Middle and Bottom). Microwave processing time was varied as $30,60,90,120,150,180,210$ Seconds. Their results revealed effectiveness of the microwave heating over the conventional heating in breaking w/o emulsions. Vladan Rajakovic et al. have used the combined Freeze/Thaw and microwave irradiation method to spilt oil from oil-in-water emulsion that was originated from metal working oil. The splitting process was carried out via the ultrafreezer device at three distinct temperatures $-20,-40$ and $-60^{\circ} \mathrm{C}$. The sample was first freezed $10 \mathrm{~h}$, then thawed back in Air at $20^{\circ} \mathrm{C}$, then water bath at $40^{\circ} \mathrm{C}$ or microwave at $95^{\circ} \mathrm{C}, 800 \mathrm{w}, 2450 \mathrm{MHz}$. Their results showed that, the effectiveness of the freeze/thawing and microwave demulsification techniques depend mainely on the oil content of the emulsion sample, freezing time, thawing steps, and thawing time. Indeed, microwave also believed to induce molecules to acquire more energy because of super heating, and volumetric heating. Therefore, microwave demulsification is proved to be the most effective method in breaking emulsions [73]. Nour et al. have investigated the batch microwave oven with $2450 \mathrm{MHz}$ frequency in treating and breaking w/o emulsion that was stabilised by some commercially available emulsifiers. The commercial emulsifiers used in this study were Triton-X100 and Low Sulfur Wax Residu (LSWR). Their experimental emulsion was consisted of 20-80 and 50-50\% water to oil ratios. The crude used was given by PETRONAS (Malaka refinery). The mixing speed was fixed as $1800 \mathrm{rpm}$ for $8 \mathrm{~min}$. However, their comprehensive study have proved that, microwave can provide very fast and uniform heating and have a very strong potential to be used as an alternative demulsification technique for petroleum emulsion treatment [74]. Abdulbari et al. have studied the stability as well as microwave demulsification of petroleum emulsion. Various commercial emulsifiers used, including, Span 83, Triton-x100, Low sulfur wax residue (LSWR), and sodium dodecyl sulfate (SDDS). the targeted parameters were, the surfactant types, surfactants concentration, water volume fractions in the emulsion (10-90\%), emulsification temperature and agitation speed. The raw crude petroleum oil were originated from Iran and Malaysian oil fields. Experimental emulsions were prepared through the agent in oil method. The emuksification speed was $1600 \mathrm{rpm}$. The demulsification experiment was carried out using the elba microwave oven whose power output was $900 \mathrm{w}$, and frequency of $2450 \mathrm{MHz}$. their results showed that Microwave gave rapid separation over the conventional gravitational settling [75]. Montserate Fortuny et al. have investigated the important parameters for microwave demulsification, namely, salinity, temperature, water content and $\mathrm{pH}$. Their experimental emulsion was prepared by mixing the crude oil with saline water, and homogenized using two steps emulsification techniques. In the first step, the mixture was mixed by simple hand shaking and certain amount of water was added in a drop-wise fashion while the mixing is going on. In the second step, the system was vigorously mixed using the ultra-turax T-25 homogenizer fitted with a S25-25G dispersing tool. The water volume fraction was varied as $(25,35,45 \%)$. Emulsion were mixed at $17500 \mathrm{rpm}$ for $10 \mathrm{~min}$ respectively. The demulsification experiment were performed using the commercial microwave, at processing time of $15 \mathrm{~min}$. Results showed that, increasing microwave power could increase the demulsification process, beside that, high water contained emulsions, were found to give high separation efficiency and that is because of the dielectric heating. Also high salt content or high $\mathrm{pH}$ had reduced the demulsification rate. Indeed, the best separation rate was observed with emulsion having water volume fraction of $45 \%$ and $\mathrm{pH}$ of 7 [76].

Yunus et al. have conducted a laboratory experiment to examine the performance of microwave heating technique in breaking water-incrude oil emulsion in comparison with the conventional demulsification techniques, and their results had concluded that, microwave gave better separation than the conventional heating. They claimed that, microwave does not need any chemicals addition, and could be used as an alternative green technique to treat petroleum emulsions [77].

Ilia Anisa et al. have also used the microwave demulsification techniques to treat w/o emulsions, and optimize the operating conditions to fix the best operating conditions for microwave demulsification by using the RSM software [75,78]. Lixin Xia et al. have studied the stability of crude oil emulsion and its relation to the presence of Asphaltene and resins (the natural surfactants), followed by microwave demulsification. Their experimental emulsion was produced by using jet kerosene as model oil phase mixed with certain amounts of asphaltene and resin. The asphaltene and resin were extracted from Daqing crude oil field (China). The concentration of the asphaltene in the bulk emulsion was varied in the range of 0.3 to $0.9 \%(\mathrm{w} / \mathrm{w})$, and that of the resin was in the range of $1-4 \%(w / w)$. Emulsion was prepared by simple hand shaking. The demulsification process was carried out by using either oil bath at $90^{\circ} \mathrm{C}$, or microwave oven with power output frequency of $850 \mathrm{w}$ and $2450 \mathrm{MHz}$ respectively. Their results revealed that, stability was strongly depended on the amount of the asphaltene and resins in the daqing crude oil, and that is because the adsorption of these interfacial active components at the interfaces is believed to form a mechanical film between the droplets, also microwave demulsification had given better separation result compared to the conventional demulsification methods. Abdurahman et al. have proposed a continuous microwave demulsification method for water-in-crude oil emulsion, and found that the temperature rise within the sample at given location was linear, and the rate of temperature increase of emulsion decreases at elevated temperature due to the decrease of dielectric loss of water [79].

\section{Ultrsonic demulsification}

Ultrasonic is the branch of physics that is dedicated to the studies of sounds, including, generation, transmission, control, reception as well as the effects of mechanical waves in solids, liquids and gases [80]. Ultrasonic are used in various fields, some owhich are environmental, architectural, musical and engineering acoustics. Ultrasonic or Accostic is the study of sound with a frequency higher than audible sound. Environmental acoustics deals with noise control. Architectural acoustics is the study of how sound waves and buildings interact. Musical acoustics deals with the design and use of musical instruments and how they affect the listener. Engineering acoustics concerns the recording and reproduction of sound. Thus, ultrasound are well established in most engineering application and material processing such as sonochemistry, metal working, cleaning and many more. Each 
application have certain range of frequency. The basic requirement of fabricating an ultrasonic equipment are transducer (a device that can convert electric charges to sound wave) and medium within which sound could propagate (Mostly liquids), for sonochemical application the medium is mostly water, while, transducers vary with application. In the following paragraphs some criteria and types of the existing transducers would be highlighted [81]. Gautam et al. have applied the low intensity ultrasonic field to separate oil phase that is suspended in an aqueous solution. However, the system consisted of rectangular chamber that was filled with matrix of porous medium within which the oil drops was assumed to be filled, three types of mesh were used that were glass beads, aluminum mesh and polyester. Rectangular piezoelectric (PZT) transducer and stainless steel reflector were mounted in the chamber in paralleled position to each other. and the distance between the transducer and reflector was adjusted carefully to generate standing wave on the emulsion sample that was filled in chamber (between the Transducer and reflector). The acoustic field energy was produced by energizing the transducer at $680 \mathrm{KHz}$ frequency, using a continuous sonisoidal signals generated and amplified by KROHN- HITE 2100 A signal generator, and ENI 240 L power amplifiers respectively.Their experimental oil-in-water emulsion was produced by using the soybean oil and deionized water, with $50-50 \%$ oil to water ratios. Emulsion was first agitated for one minute then sonicated for 8 minutes to produce a stable emulsion having aroud 1 to $10 \mu \mathrm{m}$ sized droples, that was further diluted prior to feeding in the acoustic chamber for demulsification to ease the visual observation of the droplets' response to the ultrasonic field. Results showed that the ultrasonic separation efficiency depends strongly on the feed flow rate, path length as well as the electrical power [82].

Riera-Franco de Sarabia et al. have reported the application of ultrasound to separate the suspended particles from fluid. The basic fundamentals behind the ultrasonic particles separation, depends normally on the material to be treated. for-instance, in the case of gas systems the very fine suspended solids particles have to be removed via the agglomeration process, whereby, particles collect together to increase their sizes, then separate out of the suspending medium, while in the liquid systems the agglomeration rate is less efficient than that of the gas phase, but generally its very useful to split and separate the fine particles, from their suspending medium [83]. Riera et al. have studied the possibility to apply the ultrasonic agglomeration process to treat suspension and separate the suspended particles from industrial fumes. They observed that, the agglomeration process was not very good in liquid systems [84]. Ye Guoxiang et al. have investigated the ultrasonic electric desalting and dewatering techniques in treating petroleum emulsions. their system was consisted of ultrasonic device and electric desalting and dewatering device that are connected in such a way that make the feed emulsion had to pass through the ultrasonic tube in order to enter the electric desalting and dewatering units. The ultrasonic equipment was designed with very specific geometry to produce an ultrasonic standing waves field. The frequency used was either 10 or $20 \mathrm{KHz}$. The initial salt content of the petroleum oil was as high as $40-70 \mathrm{mg} / \mathrm{L}$. The process was carried out in three distinct steps that are, crude oil pretreatment, ultrasonic irradiation, and electric desalting and dewatering. During pretreatment, crude oil was treated to render it less viscous and flowable, then certain amount of water was added in the oil containing demulsifiers in the pipeline, then the mixture was agitated by static agitator. In the second stage, emulsion was treated with ultrasonic standing wave field. In the third stage, emulsion was treated with electric desalting and dewatering process, and this caused the water droplets to coalesce and settle down as separate phases. Results showed that, ultrasonic electric united process increased the dewatering and desalting process of the crude oil emulsion. The optimum treatment conditions were found to be $5 \%$ by volume of injected water, $30 \mu \mathrm{g} / \mathrm{g}$ of demulsifiers, $0.45 \mathrm{MPa}$ of Mixing pressure drop, $10 \mathrm{KHz}$ and $150 \mathrm{w}$ of ultrasound (standing waves field), $3.4 \mathrm{~min}$ ultrasound irradiation time, $1.2 \mathrm{Kv} / \mathrm{cm}$ electric intensity, 20 $\mathrm{L} / \mathrm{h}$ of emulsion flow rate, and processing temperature of $80^{\circ} \mathrm{C}$. Their system was reported to be very effective, hence beside water separation, the salt concentration was also found decrease from $67.5 \mathrm{mg} / \mathrm{l}$ to 3.97 $\mathrm{mg} / \mathrm{l}$ at $80^{\circ} \mathrm{C}[85]$.

Sanjay et al. had studied the mechanism of aggregation of fine particles in porous medium under the influence of the standing waves field; the parameters of interest were, the bulk fluid flow rate, ultrasonic power, and the feed concentration. Ultrasonic device was a rectangular chamber that consisted of two rectangular leads on parallel position to each other and piezoelectric transducer. A porous mesh was placed between the transducer and reflector, their results showed that the separation efficiency had reached up to $70-80 \%$ by using the porous media. Separation efficiency was found to be directly proportional to the flow rate of the suspension, while the particle concentration was observed to have no effect on the separation efficiency [85].

Nii et al. have used a $2 \mathrm{MHz}$ ultrasonic frequency generating devices to treat emulsions that were originally prepared from canola oil and water. The parameters investigated were the ultrasonic power and processing time. However, their experimental emulsion was produced by mixing deionized water and canolla oil, mixing power was supplied by an ultrasonic horn sonifier (BRANSON DIGITAL). The demousification experiment was carried out by doting a drop from the stable emulsion on microscopic lens, then the lens was soaked in an ultrasonic water bath that was consisted of glass cylinder with stainless steel plate at the bottom and transducer was mounted on the steel plate. Results proved that, the action of the ultrasonic power pushed the oil droplets and lead them to aggregation and eventually to massive coalescence and phase separation. beside that a remarkable increase in the velocity of the oil droplets was observed after sample was irradiated via the ultrasonic [86]. Garcia et al. have applied the ultrasonic standing wave resonanting chamber to treat $\mathrm{o} / \mathrm{w}$ emulsions. They have used an ultrasonic frequency range of between $1-2 \mathrm{MHz}$, and the apparatus used was standard research system ENI 310L RF power amplifier Tektronix oscilloscop, the experimental emulsion used in their study was prepared using an Omni-Ruptor 250 Homogenizer to provide the mixing power. The oil volume fraction in the emulsion was fixed at $10 \%$. For demulsification experiment, several transducer types were examined to generate the standing wave in the emulsion. However, once the standing wave was generated, The oil droplets were observed to aggregate as response to the effect of the standing waves, and thus the standing wave treated $\mathrm{o} / \mathrm{w}$ emulsion sample had shown a great separation efficiency over the control or the gravitationally separating sample. It was also observed that, at elevated ultrasonic power, acoustic streaming and heating could be produced. Furthermore, directly contacting the emulsion sample with the transducer was found to be very effective [87].

\section{Summary}

Crude petroleum is almost always exists in the forms of emulsions of various types including, w/o, o/w, w/o/w, o/w/o. With w/o being the most prominent type encountered in petroleum institutions, that is imparted to the fact that most of the indigenous surface active agents such as asphaltene, resin, long chain fatty acids are oil soluble 
amphiphilics. Water comes into contact with petroleum oil either inside the well, or during pumping from the reservoir, as well as during desalting process, this latter is very important in petroleum industries wherein water is used as a cost effective solvent to remove the water soluble salts from the petroleum prior to refining process. Indeed water also can be used to reduce the viscosity in the case of heavy oil transportation in pipelines.However, the presence of water in the crude petroleum causes other problems such as corrosion, quality reduction of the refined products. Therefore, water must be removed or reduced to minimum amount before refining would start. Various demulsification techniques are currently used to remove water from crude oil, including chemicals, conventional heating, electrical and centrifugal, with chemical being the most prominent and widely used, but as reported in literature, it has a lot of disadvantage in many aspects, including, safety, cost and environmental effect, therefore, more and more versatile, lucrative and sustainable techniques are needed, and that is where the objective of the current work comes in. Moreover, emulsion formation and stability varies from one location to another, even between two different wells in the same field, Currently chemical demulsification is the most dominating technique in action. In chemical demulsification, Amphiphilic copolymers called demulsifiers that categorized into anionic, cationic, nonionic and ziwitrionic are injected to the emulsion with respect to their major groups. Microwave demulsification have gained a considerable attention in resent years. it was first introduced to this field of petroleum emulsion by Wolf in his various lab scale experiments. Recently the application of ultrasonic in crude oil teatment was also reported. Most researches in crude emulsion were performed in experimental emulsion that mimic the real emulsions in their characteristics. However, there are some chalenges in prepaering the suitable experimental emulsion, since every researcher uses his own formultion and characters. Table 1 below reveals some of the formulation and parameters used to produce stable experimental petroleum based emulsions.

\section{Conclusions and Recomendations}

This essay had surveyed the ultimate techniques and methods of preparing and treating crude oil emulsions and water separation (demulsification). Based on chemical demulsification, polysiloxane demulsifiers claimed to be very efficient and environmental friendly, compared to the commercial demulsifiers. However, they have not being used because of economical reason. Microwave and ultrasonic techniques were found to be very effective in separating water from petroleum. Microwave is commonly preferred in material processing over its conventional counter part, due to its volumetric as well as dielectric heating. The mechanism of heating in conventional heating occurs through diffusion of heat from the surface of the material to the bulk, which the gradient in heating rate. whereas, in microwave heating, the temperature gradient in different location within the sample is almost invariant, that means microwave heat materials uniforly and volumetricaly. another important phenomenon is that different material possess different heating pattern, and that is due to the variation in material's microwave absorption capacity, which in turn depends on dielectric properties. Both microwave and ultrasonic are more environmental friendly and costeffective, but have not beeing commercialized yet. Other minor techniques such as, centrifuge, membrane and bacterial treatment also reported in the literature.

As mentioned earlier, chemical demulsification is not really recomended from environmental view point, hence some suggstion are given next:

- Developing more green chemical that is cost effective, and environmental friendly

- Commercialization of microwave demulsification process to operate in mass treatment of petroleum

- Developing the ultrasonic techniques for petroleum treatment

\begin{tabular}{|c|c|c|c|c|c|c|}
\hline No & Oil type & Surfactants & Mixture type & $\begin{array}{l}\text { RPM } \\
\left(S^{-1}\right)\end{array}$ & $\begin{array}{l}\text { Mixing time } \\
\text { (minutes) }\end{array}$ & Reference \\
\hline 01 & Heptane/toluene & Asphaltene/resin & Ultra-turax T25 & 9500 & 0.5 & Havre and Sjöblom [5] \\
\hline 02 & Jet Kerosen & Asphaltene/resin & - & - & - & - \\
\hline 03 & Silicon oil & SDS & - & 7000 & - & Burjic [7] \\
\hline 04 & Heptane/toluene & Asphaltene/resin & Ultra-turax T25 & 22000 & 30 & Andreas et al. [13] \\
\hline 05 & Heptane/toluene & Asphaltene/resin & - & 17000 & 7 & Rodiguez et al. [12] \\
\hline 06 & Paraffin oil & Span 80,Tween20 & - & 500 & 15 & Zhang et al. [16] \\
\hline 07 & - & - & & 10000 & 30 & Fara et al. [19] \\
\hline 08 & Petroleum oil & - & Ultra-turax & 24000 & 2 & Paso et al. [21] \\
\hline 09 & Bitumen/toluene & - & Polytron & 20000 & 3 & Wu et al. [25] \\
\hline 10 & - & SDDS & Ultrasonic & - & 10 & Bratskaya et al. [30] \\
\hline 11 & Petroleum oil & Silverson lurt & - & 20000 & 1 & Auflem [32] \\
\hline 12 & - & - & Vertishear cyclone & 1500 & 2 & Zaki et al. [4] \\
\hline 13 & Petroleum oil & - & ht-2 homogenizer & 1200 & 5 & Zhiqing [33] \\
\hline 14 & Petroleum oil & - & 3 blade propeller & 2000 & 10 & Nour [34] \\
\hline 15 & Benzene & SDDS & Magnatis stirrer & - & 15 & Yakhkeshi and Hosseina [46] \\
\hline 16 & Xelene & - & Ultrasonic & - & 6 & Oji and Opara [47] \\
\hline 17 & - & - & Electromagnetic & 1000 & 10 & Yun Kim [48] \\
\hline 18 & Petoleum oil & - & 3 blade propeller & 1600 & 5 & Nour [34] \\
\hline 19 & - & Span 80 & - & 6000 & 20 & Lin et al. [54] \\
\hline 20 & Dodecane & Asphaltene & Hand shaking & - & & Yang et al. [55] \\
\hline 21 & - & Twee 20 & blender & & & Mittal and Vold [57] \\
\hline 22 & Kerosen & - & - & 1000 & 60 & Kocherginsky et al. [58] \\
\hline 23 & Gas oil & - & 6 blade blender & - & 10 & Sadrzadeh et al. [62] \\
\hline 24 & - & - & Ultra-turax T25 & 17500 & 10 & Fortuny et al. [71] \\
\hline
\end{tabular}

Table 1: Some importants parameters, for emulsion formulation. 
Citation: Issaka SA, Nour AH, Yunus RM (2015) Review on the Fundamental Aspects of Petroleum Oil Emulsions and Techniques of Demulsification J Pet Environ Biotechnol 6: 214. doi:10.4172/2157-7463.1000214

- Integration of the above mentioned techniques

- Combining chemical with microwave or ultrasound to reduce the dosage of chemicals.

\section{References}

1. Kristiansen TS, Lewis A, Daling PS, Nordvik AB (1995) Heat and chemical treatment of mechanically recovered w/o emulsions. Spill Science and technology 2: 133-141.

2. Auflem IH, Kallevik H, Westvik A, Sjoblom J (2001) Influence of pressure and solvency on the separation of water-in-crude-oil emulsions from the North Sea. Petroleum Science and Engineering 31: 1-12.

3. David DD, Pezron I, Dalmazzone C, Noık C, Clausse D, et al. (2005) Elastic properties of crude oil/water interface in presence of polymeric emulsion breakers. Colloids and Surfaces A: Physicochem Eng Aspects 270: 257-262.

4. Zaki NN, Carbonell RG, Kilpatrick PK (2003) A Novel Process for Demulsification of Water-in-Crude Oil Emulsions by Dense Carbon Dioxide. Ind Eng Chem Res 42: $6661-6672$.

5. Havre TK, Sjöblom J (2003) Emulsion stabilization by means of combined surfactant multilayer (D-phase) and asphaltene particles. Colloids and Surfaces A: Physicochem Eng Aspects 228: 131-142.

6. Xia L, Lu S, Cao G (2004) Stability and demulsification of emulsions stabilized by asphaltenes or resins. Colloid and Interface Science 271: 504-506.

7. Burjic $\mathrm{J}$ (2003) Measuring the distribution of interdroplet forces in a compressed emulsion system. Physica A 327: 201-212.

8. Guo J, Liu Q, Li M, Wu Z, Christy AA (2006) The effect of alkali on crude oil/ water interfacial properties and the stability of crude oil emulsions. Colloids and Surfaces A: Physicochem Eng Aspects 273: 213-218.

9. Li M, Lin M, Wu Z, Christy AA (2004) The influence of $\mathrm{NaOH}$ on the stability of paraffinic crude oil emulsion. Fuel 84: 183-187.

10. Kumar K, Nikolov AD, Wasan DT (2001) Mechanisms of Stabilization of Waterin-Crude Oil Emulsions. Ind Eng Chem Res 40: 3009-3014.

11. Aske N, Kallevik H, Blom JS (2002) Water-in-crude oil emulsion stability studied by critical electric field measurements. Correlation to physicochemical parametersand near-infrared spectroscopy. Petroleum Science and Engineering 36: 1-17.

12. Valverde MARG, VI'Ichez MAC, Duenas AP, Ivarez RHA (2003) Stability of highly charged particles: bitumen-in-water dispersions. Colloids and surfacees: physicochem Eng Aspects 222: 233-251.

13. Hannisdal A, Ese MH, Hemmingsen PV, Oblom JS (2006) Particle-stabilized emulsions: Effect of heavy crude oil components pre-adsorbed onto stabilizing solids. Colloids and Surfaces A: Physicochem. Eng. Aspects $276: 45-58$.

14. Moran K, Czarnecki J (2007) Competitive adsorption of sodium naphthenates and naturally occurring species at water-in-crude oil emulsion droplet surfaces. Colloids and Surfaces A: Physicochem Eng Aspects 292: 87-98.

15. Quintero CG, Noïk C, Dalmazzone C, Grossiord JL (2008) Modelling and characterisation of diluted and concentrated water-in- crude oil emulsions: comparison with classical behavior. Rheol Acta 47: 417-424.

16. Zhang L, Que G (2008) Influence of the HLB parameter of surfactants on the dispersion properties of brine in residue. Colloids and surfaces $A$ : physiochemical and engineering aspects 320 : 111-114.

17. Bratskaya S, Avramenko V, Schwarz BS, Philippova I (2006) Enhanced flocculation of oil-in-water emulsions by hydrophobically modified chitosan derivatives. Colloids and Surfaces A: Physicochem Eng Aspects 275: 168-176.

18. Li M, Xu M, Ma Y, Wu Z, Christy AA (2001) The effect of molecular parameters on the stability of water-in-crude oil emulsions studied by IR and UV spectroscopy. Colloids and Surfaces A: Physicochemical and Engineering Aspects 197: 193-201.

19. Farah MA, Oliveira RC, Caldas JN, Rajagopal K (2005) Viscosity of water-in-oil emulsions: Variation with temperature and water volume fraction. Journal of Petroleum Science and Engineering 48: 169-184

20. Sanfeld A, Steinchen A, Mishchuk (2005) Energy barrier in dense W/O emulsions. Colloids and Surfaces A: Physicochem Eng Aspects 261: 101-107.

21. Paso K, Silset A, Sorland G (2009) Characterization of the Formation,
Flowability, and Resolution of Brazilian Crude Oil Emulsions. Energy and Fuels 23: $471-480$

22. Dicharry C, Arla D, Sinquin A, Graciaa A, Bouriat $P$ (2006) Stability of water/ crude oil emulsions based on interfacial dilatational rheology. Colloid and Interface Science 297: 785-791.

23. Johnsen EE, Rønningsen HP (2003) Viscosity of 'live' water-in-crude-oil emulsions: experimental work and validation of correlations. Petroleum Science and Engineering 48: 169-184.

24. Kukizaki M, Goto M (2008) Demulsification of water-in-oil emulsions by permeation through Shirasu-porous-glass (SPG) membranes. Journal of Membrane Science 32: 196-203.

25. Wu J, Xu Y, Dabros T, Hamza H (2004) Effect of EO and PO positions in nonionic surfactants on surfactant properties and demulsification performance. Colloids and Surfaces A: Physicochem. Eng. Aspects 252: 79-85.

26. Kang W, Jing G, Li HZM, Wu Z (2006) Influence of demulsifier on interfacial film between oil and water. Colloids and Surfaces A: Physicochem. Eng. Aspects 272: $27-31$.

27. Hafiz AA, El-Din HM, Badawi AM (2005) Chemical destabilization of oil-in-wate emulsion by novel polymerized diethanolamines. J Colloid Interface Sci 284 167-175.

28. Alejandro AP, George J, Hirasaki, Miller CA (2005) Chemically Induced Destabilization of Water-in-Crude Oil Emulsions. Ind Eng Chem Res 44: 1139 1149 .

29. Dalmazzone C (2005) Mechanism of crude oil-interface destabilization by silicone demulsifiers. SPE International symposium on oilfield chemistry SPE 80241

30. Bratskaya S, Avramenko V, Schwarz S, Philippova I (2006) Enhanced flocculation of oil-in-water emulsions by hydrophobically modified chitosan derivatives. Colloids and Surfaces A: Physicochem. Eng. Aspects 275: 168-176.

31. Ezzati A, Gorouhi E, Mohammadi T (2005) Separation of water in oil emulsions using microfiltration. Desalination 185: 371-382.

32. Auflem IH (2002) Influence of asphaltene aggregation and pressure on crude oil emulsion stability, PhD thesis, Department of chemical engineering, Norwegian University of science and technology, Trondheim

33. Zhiqing Z (2004) Characterisation and demulsification of poly (ethylene oxide) block-poly (propylene oxide)-block-poly (ethylene oxide) copolymers. Colloidal and Interface Science 777: 464-470.

34. Nour AH (2009) Demulsification of Virgin coconut oil by centrifugation method: A feasibility study. International Chemical Technology 1: 59-64.

35. Yang CL (2007) Electrochemical coagulation for oily water demulsification Separation and Purification Technology 54: 388-395

36. Harpur G (1997) Destabilization of water-in-crude oil emulsions under the influence of an $\mathrm{AC}$ electric field: experimental assessment of the performance. Electrostat 40: 135-140.

37. Jang W, Lee $Y(2000)$ Removing oil from oil-in-water emulsion using electrical demulsification method. Industrial and Engineering Chemistry 6: 85-92.

38. Sams GW, Zaouk M (2000) Emulsion Resolution in Electrostatic process Energy and fuel 14: 31-37.

39. Ichikawa T, Itoh K, Yamamoto S, Sumita M (2004) Rapid demulsification of dense oil-in-water emulsion by low external electric field :I. Experimenta evidence. Colloids and Surfaces A: Physicochem Eng Aspects 242: 21-26.

40. Fordedal H, Sjoblom J (1996) Percolation Behavior in W/O Emulsions Stabilized by Interfacially Active Fractions from Crude Oils in High External Electric Fields. Colloid and interface science 181: 589-594.

41. Yoshida J, Chen J, Aoki K (2003) Electrochemical coalescence of nitrobenzene j water emulsions. Electroanalytical Chemistry 553: 117-124.

42. Bailesa PJ, Kuipa PK (2001) The efect of air sparging on the electrical resolution of water-in-oil emulsions. Chemical Engineering Science 56: 6279-6284.

43. Ichikawa T (2007) Electrical demulsification of oil-in-water emulsion. Colloids and Surfaces A: Physicochem Eng Aspects 302: 581-586

44. Kanazawa S, Takahashi Y, Nomoto Y (2008) Emulsification and Demulsification Processes in Liquid-Liquid System by Electrostatic Atomization Technique. Industry applications, IEEE transactions 44: 1084-1089. 
Citation: Issaka SA, Nour AH, Yunus RM (2015) Review on the Fundamental Aspects of Petroleum Oil Emulsions and Techniques of Demulsification J Pet Environ Biotechnol 6: 214. doi:10.4172/2157-7463.1000214

45. Haifeng G, Ye P (2008) Polarization Characteristic of Droplet of Water-in-Oil Emulsion in a High Uniform Electric Field. International Workshop on Modelling, Simulation and Optimization 139-142.

46. Yakhkeshi A, Hosseina M (2010) Demulsification of Benzene-in-water emulsion by electric field. Middle east Journal of scientific Research 5: 57-60.

47. Oji A, Opara CC (2012) Electrocoalescence of Field Crude Oil using High voltage Direct Current. International Journal of Engineering Science and Technology.

48. Yun Kim B (2007) Demulsification of water-in-crude oil emulsions by a continuous electrostatic dehydrator. Separation Science and Technology 37 : 1307-1320.

49. Dalling S (2003) Norwegian testing of emulsion properties at sea-the importance of oil type and release conditions. Spill science and technology Bulletin 8: 123-323.

50. Gunet CC, Sveum (1995) In situ burning of emulsions R\&D in Norway. Spil science and technology Bulletin 2: 75-77.

51. Binks BP, Rocher A (2009) Effects of temperature on water-in-oil emulsions stabilised solely by wax Microparticles. Colloid and Interface Science 335: 94-104.

52. Taylor SE (2011) Thermal destabilisation of bitumen-in-water emulsions-A spinning drop tensiometry study. Fuel 90: 3028-3039.

53. Chen G, He G (2003) Separation of water and oil from water-in-oil emulsion by freeze/thaw method. Separation and Purification Technology 31: 83-89.

54. Lin C, He G, Li X, Peng L, Dong C, et al. (2007) Freeze/thaw induced demulsification of water-in-oil emulsions with loosely packed droplets. Separation and Purification Technology 56: 175-183.

55. Yang X, Tan W, Yu Bu (2009) Demulsification of Asphaltenes and Resins emulsions via freeze/thaw method. Energy and fuels 23: 481-486.

56. Habn AU, Mittal KL (1979) Mechanism of demulsification of oil-in-water emulsion in the centrifuge. Colloid and polymer sci 257: 959-967.

57. Mittal KL, Vold RD (1972) Effects of initial concentration of emulsifying agent on the centrifugal stability of oil-in-water emulsions. American oil chemistry society 491: $527-532$.

58. Kocherginsky NM, Tan CL, Lub WF (2003) Demulsification of water-in-oil emulsions via filtration through a hydrophilic polymer membrane. Membrane Science 220: 117-128

59. Nadarajah N, Singh A, Ward OP (2002) Evaluation of a mixed bacterial culture for de-emulsification of water-in-petroleum oil emulsions. World Journal of Microbiology and Biotechnology 18: 435-440.

60. Dantas TNC, Neto AAD, Moura EF (2001) Microemulsion systems applied to breakdown petroleum emulsions. Petroleum Science and Engineering 32: 145-149.

61. Colle RD, Longo E, Fontes SR (2007) Demulsification of water/sunflower oil emulsions by a tangential filtration process using chemically impregnated ceramic tubes. Membrane Science 289: 58-66.

62. Sadrzadeh M, Gorouhi E, Mohammadi T (2008) Oily wastewater treatment using polytetrafluoroethylene (PTFE) hydrophobic membranes. Twelfth International Water Technology Conference.

63. Lozsan A (2012) Salt-induced fast aggregation of nano emulsions: structural structural and kinetic scaling. Colloid and Polymer Science 290: 1561-1566.

64. Thostenson, Chou (1999) Microwave processing: fundamentals and applications. Composite A 30: 1055-1071.

65. Camelia (1998) Dielectric parameters relevant to microwave dielectric heating Chemical Society Reviews 27: 213-224.

66. Barringer (1994) Effect of sample size on the microwave heating rate: oil vs Water. AIChE journal 40: 1433-1439.

67. Montserrate (2007) Effect of Salinity, Temperature, Water Content, and pH on the Microwave Demulsification of Crude Oil Emulsions. Energy and Fuels 21: 1358-1364.

68. Helene (2009) Dielectric properties of crude oil components. Energy Fuels 23 5596-5602.

69. Samanta SK, Basak T, Sengupta B (2008) Theoretical analysis on microwave heating of oil-water emulsions supported on ceramic, metallic or composite plates. International Journal of Heat and Mass Transfer 51: 6136-6156.
70. Dicharry C, Arla D, Sinquin A, Graciaa A, Bouriat P (2006) Stability of water/ crude oil emulsions based on interfacial dilatational rheology. Journal of Colloid and Interface Science 297: 785-791.

71. Fortuny M, Oliveira CBZ, MeloRLFV, Nele M (2007) Effect of Salinity, Temperature, Water Content, and $\mathrm{pH}$ on the Microwave Demulsification of Crude Oil Emulsions. Energy Fuels 21: 1358-1364.

72. Bjorendalen N (2004) The effect of microwave and ultrasonic irradiation on crude oil during production with a horizontal well. Petroleum science and engineering 43: 139-150.

73. Rajakovic V, Skala D (2006) Separation of water-in-oil emulsions by freeze/ thaw method and microwave radiation. Separation and Purification Technology 49: $192-196$

74. Nour AH (2010) Demulsification of water-in-crude oil (w/o) emulsions by using Microwave radiations. Journal of Applied sciences 10: 2935-2939.

75. Anisa ANL, Nour AH (2011) Destabilization of heavy and light crude oil emulsions via Microwave heating Technology: An optimization study. Journa of Applied Science 11: 2898-2906.

76. Nour AH, Yunus RM (2006) A continuous microwave heating of water-in-crude oil emulsions: An experimental stud. Journal of Applied science 6: 1868-1872.

77. Yunus RM, Nour AH (2005) Water-in-oil demulsification via microwave irradiation. Proceedings of the International Conference on Recent Advances in Mechanical \& Materials Engineering 30-31.

78. Nour AH, Anisa ANI (2012) Demulsification of water-in-oil (W/O) emulsion via microwave irradiation: An optimization. Journal of Scientific Research and Essays 7: 231-243.

79. Abdulbari HA, Abdurahman NH, Rosli YM, Mahmood WK, Azhari HN (2011) Demulsification of petroleum emulsions using microwave separation method International Journal of the Physical Sciences 6: 5376-5382.

80. Nguyen NT (2005) Wu ZG Micro mixers : A review. Micromech Microeng 151-16

81. Mason TJ, Lorimer JP (2002) Use of power ultrasound in chemistry and processing. Journal of Applied sonochemistry.

82. Pangu GD, Feke DL (2004) Acoustically aided separation of oil droplets from aqueous emulsions. Chemical Engineering Science 59: 3183- 3193.

83. Sarabia ER, Gallego-Jua'rez JA, Corral GR, Segura LE (2000) Application of high-power ultrasound to enhance fluid/solid particle separation processes. Journal of Ultrasonics 38: 642-646.

84. Guoxiang YE, Xiaoping LU, Peng F, Pingfang H, Xuan S (2008) Pretreatment of Crude Oil by Ultrasonic-electric United Desalting and Dewatering. Chinese Journal of Chemical Engineering 16: 564-569.

85. Gupta S, Feke DL (1997) Acoustically driven collection of suspended particles within porous media. Journal of Ultrasonics 35: 131-139.

86. Nii S, Kikumoto S, Tokuyama H (2008) Quantitative approach to ultrasonic emulsion separation. Ultrasonics Sonochemistry 1: 145-149.

87. Lopez A, Gand Sinha DN (2008) Enhanced Acoustic Separation of Oil-Water Emulsion in Resonant Cavities, The Open Acoustics Journal 1: 66-71. 